\title{
TRMM PR Standard Algorithm 2A23 and its Performance on Bright Band Detection
}

\author{
Jun AWAKA \\ Department of Marine Biology and Sciences, Tokai University Sapporo Campus, Sapporo, Japan
}

Toshio IGUCHI

National Institute of Information and Communications Technology (NICT), Applied Electromagnetic Research Center, Koganei, Japan

and

Ken'ichi OKАMOTO

Faculty of Environmental and Information Studies, Tottori University of Environmental Studies, Tottori, Japan

(Manuscript received 13 June 2008, in final form 3 February 2009)

\begin{abstract}
This paper presents the statistical properties of bright band (BB) measured by the Tropical Rainfall Measuring Mission (TRMM) Precipitation Radar (PR). Since BB is detected by PR standard version 6 (V6) algorithm 2A23 V6, this paper first outlines $2 \mathrm{~A} 23 \mathrm{~V} 6$, which classifies rain into three main categories: stratiform, convective, and other.

This paper studies BB from the viewpoint of algorithm development. It demonstrates that the detected BB count strongly depends on the antenna scan angle. The BB height (HBB) detected by the PR is compared with the $0^{\circ} \mathrm{C}$ height computed using the National Centers for Environmental PredictionDepartment of Energy Atmospheric Model Intercomparison Project-II reanalysis (NCEP2) data. On average, the BB height is about $500 \mathrm{~m}$ below the $0^{\circ} \mathrm{C}$ height, which is computed from the NCEP2 data. However, a large difference between the above two heights is sometimes observed, mainly due to the false detection of BB. The reliability of BB detection by the current PR algorithm 2A23 V6 could be increased by introducing a simple filter that rejects BBs by regarding them as false when the number of BBs detected in one antenna scan (consisting of 49 antenna beam directions) is less than or equal to 5 . An improved version of the filter is planned to be installed in the coming 2A23 V7. Statistics of 10 years of PR data indicate a small but discernible effect of the August 2001 boost of the altitude of the TRMM satellite on the rain type statistics and on the BB statistics. The zonal mean of the maximum value of reflectivity factor in the $\mathrm{BB}$ peak $(\mathrm{ZmaxBB})$ over water is almost constant in latitude, but the zonal mean of $\mathrm{ZmaxBB}$ over land is less at latitudes above 20 degrees in the northern hemisphere. The width of $\mathrm{BB}$ increases as ZmaxBB increases. The zonal mean of the BB width in mid latitude exhibits a small but smooth decrease as the latitude increases.
\end{abstract}

Corresponding author: Jun Awaka, Department of Marine Biology and Sciences, Tokai University Sapporo Campus, Minami-ku, Minami-sawa 5-1-1-1, Sapporo 005-8601, Japan

E-mail: awaka@tspirit.tokai-u.jp

(C)2009, Meteorological Society of Japan

\section{Introduction}

A bright band (BB), i.e., a melting layer, where the solid particles aloft melt and change to raindrops (e.g., Battan 1973; Meneghini and Kozu 1990), is important to both meteorology and radio engineering (Thurai et 


\section{al. 2005).}

The BB appears in the case of stratiform rain (e.g., Steiner et al. 1995; Bringi and Chandrasekar 2001) as a strong peak of radar echo when the radar frequency is not so high, i.e., below 15 to $20 \mathrm{GHz}$. Hence, in radar observation of rain, detected BB is a good indicator of stratiform rain. BB detection can thus be used for rain-type classification.

Since the BB peak appears near the height of $0^{\circ} \mathrm{C}$ air temperature, the detected height of the $\mathrm{BB}$ peak is approximately $0^{\circ} \mathrm{C}$ height, which is important information for many areas of interest because the $0^{\circ} \mathrm{C}$ height is a boundary between solid particles and liquid particles, the latter of which appreciably attenuate radio signals at frequencies above $10 \mathrm{GHz}$.

This paper presents the statistical properties of BB as observed by the Precipitation Radar (PR) (Kozu et al. 2000) aboard the Tropical Rainfall Measuring Mission (TRMM) (Kummerow et al. 2000) satellite. Algorithm 2A23, a standard algorithm of the TRMM PR, detects BB. Algorithm 2A23 also classifies rain into three main categories: stratiform, convective, and other (Okamoto et al. 1998; Awaka et al. 2007).

The main purpose of this paper is to demonstrate how the current $2 \mathrm{~A} 23$ version 6 (V6) detects BB by using long-term PR data and to examine possible improvements of $\mathrm{BB}$ detection in the next version of 2A23. Analysis throughout this paper is thus made from the viewpoint of algorithm development.

Though more than 10 years of PR data is now available, qualities of the data slightly changed when the TRMM satellite orbit was boosted from $350 \mathrm{~km}$ to $400 \mathrm{~km}$ in August 2001 (Takahashi and Iguchi 2004). This paper demonstrates that the effect of the orbit boost on the BB detection (and on rain type classification) is small but discernible. Since the altitude of $400 \mathrm{~km}$ is planned to be used by the Global Precipitation Measurement (GPM) core satellite that will carry the Dual-frequency Precipitation Radar (DPR) (Kobayashi and Iguchi 2003), the information about the statistics on $\mathrm{BB}$ detection and on rain type classification by 2A23 will be useful to the GPM DPR.

The greatest issue in detecting BB by $2 \mathrm{~A} 23$ is estimating the $0^{\circ} \mathrm{C}$ height. In the current $2 \mathrm{~A} 23 \mathrm{~V} 6, \mathrm{BB}$ is detected in a BB search window whose center is the $0^{\circ} \mathrm{C}$ height estimated from a climatological data set of temperature at sea level (SL), i.e., at the height of sea surface (Awaka et al. 2007).

Since the climatological temperature data at $\mathrm{SL}$ are used to detect $\mathrm{BB}$, the $\mathrm{BB}$ detected by $2 \mathrm{~A} 23$ must be validated. The use of climatological temperature data for detecting $\mathrm{BB}$ could be validated by comparing the $\mathrm{BB}$ height (HBB) detected by $2 \mathrm{~A} 23$ with the $0^{\circ} \mathrm{C}$ height calculated from reanalysis data.

Harris et al. (2000) compared HBB detected by $2 \mathrm{~A} 23$ version 5 (V5) with the $0^{\circ} \mathrm{C}$ height computed using the reanalysis data from the National Centers for Environmental Prediction (NCEP). Yamamoto et al. (2006) compared HBB detected by 2A23 V5 and the $0^{\circ} \mathrm{C}$ height com-puted using the NCEPDepartment of Energy (NCEP-DOE) Atmospheric Model Intercomparison Project-II (AMPI-II) reanalysis data (hereafter NCEP2 for simplicity), and raised a question about HBB detected by the TRMM PR in the winter season over the sea of Japan and the Yellow sea.

This paper directly compares individual HBB data detected by $2 \mathrm{~A} 23 \mathrm{~V} 6$ and the NCEP 2 derived $0{ }^{\circ} \mathrm{C}$ height by using a two-dimensional histogram and examines a possible improvement of the BB detection in $2 \mathrm{~A} 23 \mathrm{~V} 6$.

The latter part of the paper focuses on BB analysis using the 10 years of data of 3A25 V6. Algorithm $3 \mathrm{~A} 25$ is a level 3 standard TRMM PR algorithm and calculates various monthly statistics of the level 2 PR products (Okamoto et al. 1998). As mentioned above, one of the main purposes of this paper is to demonstrate how 2A23 V6 detects BB using long-term statistical data. Algorithm 3A25 V6 is very useful for this purpose.

The remaining part of this paper is organized as follows.

Section 2 first outlines 2A23 and BB detection. TRMM PR characteristics are also briefly described.

The main topics of Section 3 are as follows. Section 3.1 describes the angle-bin dependence of BB count together with those of rain type counts, and discusses the effect of the boost on the count statistics. Section 3.2 discusses estimating $0^{\circ} \mathrm{C}$ height and suggests a possible use of reanalysis data for estimating the $0^{\circ} \mathrm{C}$ height in the next $2 \mathrm{~A} 23$ version 7 (V7). Section 3.3 shows the BB statistics using 3A25 data. Section 3.4 discusses the width of BB.

Section 4 summarizes the paper.

\section{Algorithm 2A23 and BB}

\subsection{Outline of $2 A 23$}

The main objectives of PR algorithm 2A23 are to detect $\mathrm{BB}$ and classify rain types (Okamoto et al. 1998; Awaka et al. 2007). Since the rain type is needed for calculating rainfall rate in a radar observation 
of rain (e.g., Battan 1973; Meneghini and Kozu 1990), the outputs of 2A23 are used by algorithm 2A25, where rainfall rate is calculated (Iguchi et al. 2000).

Table 1 lists the TRMM PR characteristics relevant to 2A23. The TRMM satellite altitude was boosted from $350 \mathrm{~km}$ to a more air-drag free altitude of 400 $\mathrm{km}$ in August 2001 (Takahashi and Iguchi 2004) to conserve fuel and hence extend TRMM's period of service. Hereafter, we simply call this altitude change the boost. The PR is a pulsed single-frequency radar operating at $13.8 \mathrm{GHz}$ (Kozu et al. 2001). The normal range resolution is $250 \mathrm{~m}$, but over-sampled data with a $125 \mathrm{~m}$ range interval are also available near nadir angle bins. The angle bin specifies the antenna scan angle; angle-bin number 25 corresponds to the nadir direction, and the angle-bin numbers 1 and 49 to the scan edges. At the scan edges, each antenna scan angle, i.e., the off-nadir angle, is about $\pm 17^{\circ}$. The horizontal resolution of PR on the surface, that is the footprint of the PR antenna beam, was $4.3 \mathrm{~km}$ before the boost and is $5.0 \mathrm{~km}$ after the boost. The remaining items in Table 1 are self-explanatory.

TRMM PR can classify rain type by examining a three-dimensionally observed radar-reflectivity factor (Z). Amitai (1999) proposed a TRMM PR rain type classification scheme that examines the storm height and horizontal gradient of $\mathrm{Z}$ at several heights. Algorithm 2A23 classifies rain type by examining Z both vertically and horizontally (Okamoto et al. 1998; Awaka et al. 2007).

Table 1. Characteristics of TRMM PR.

\begin{tabular}{l|l}
\hline Satellite altitude & $\begin{array}{l}350 \mathrm{~km} \text { (before boost) } \\
400 \mathrm{~km} \text { (after boost) }\end{array}$ \\
Frequency & $13.8 \mathrm{GHz}$ \\
Range resolution & $250 \mathrm{~m}$ (normal sample) \\
& $125 \mathrm{~m}$ (over sample) \\
& $-17^{\circ}$ to $+17^{\circ}$ (49 angle bins) \\
Scan angles & \\
Angle bin number: & $1-49$ \\
$\quad$ Normal sample & $11-39$ (for surface) \\
Over sample & $20-30$ (for rain) \\
Over sample & $4.3 \mathrm{~km}$ (before boost) \\
Horizontal resolution & $5.0 \mathrm{~km}$ (after boost) \\
& $15 \mathrm{~km}$ above mean sea level \\
Observable range & $215 \mathrm{~km}$ (before boost) \\
Swath width & $245 \mathrm{~km}$ (after boost) \\
\hline
\end{tabular}

Altitude change was conducted in August 2001.
Figure 1 depicts a flow diagram of 2A23 V6. The input data to $2 \mathrm{~A} 23$ are from a level 1 TRMM PR algorithm called 1C21 (Okamoto et al. 1998), where the received power is converted to $\mathrm{Z}$, which includes the effect of attenuation due to precipitation, atmospheric gases, and water vapor.

Two different rain type classification methods are implemented in 2A23: a vertical profiling method (V-method) (Okamoto et al. 1998; Awaka et al. 2007) and a horizontal pattern method (H-method), which is based on the University of Washington convective/ stratiform separation method (Steiner et al. 1995).

In both methods, rain type is determined so that the rain type along a given antenna beam is the same. In other words, rain type is determined two-dimensionally in the horizontal directions. In such a case we can use a two-dimensional concept and say that the both methods classify rain type on a 'pixel' basis.

In the V-method, BB detection plays an essential part. When BB is detected along a given antenna beam, rain type for the beam is always stratiform by the V-method of 2A23 V6. (In the V-method of 2A23 $\mathrm{V} 5$, however, rain type can be classified as convective even when $\mathrm{BB}$ is detected along the antenna beam if the observed $\mathrm{Z}$ at a height well below $\mathrm{BB}$ is very strong.)

Rain types by the two methods are unified, and $2 \mathrm{~A} 23$ outputs the unified rain type, which consists of three major categories: stratiform, convective, and other. The unified rain type is also determined on a pixel basis.

Algorithm 2A23 V6 also detects shallow rain, where 'shallow' means that the storm height is $1 \mathrm{~km}$ or more lower than the estimated $0^{\circ} \mathrm{C}$ height. There are two categories of shallow rain: shallow isolated and shallow non-isolated. Shallow isolated rain is indicated when a single shallow rain pixel or connected shallow rain pixels are separated from the rain certain region (which is not shallow). Shallow non-isolated is indicated when the shallow rain is not shallow isolated. (The flow diagram of $2 \mathrm{~A} 23 \mathrm{~V} 5$ is the same as in Fig. 1 except for the part of detecting shallow rain. Only shallow isolated is detected in 2A23 V5, whereas both shallow isolated and shallow non-isolated are detected in 2A23 V6.)

The main categories of rain classified by $2 \mathrm{~A} 23 \mathrm{~V} 6$ (stratiform, convective, and other) are characterized as follows.

(1) Rain type is stratiform (1a) when BB is detected or (1b) when $\mathrm{Z}$ of the rain pixel is not strong enough to be convective. This characterization means that most 


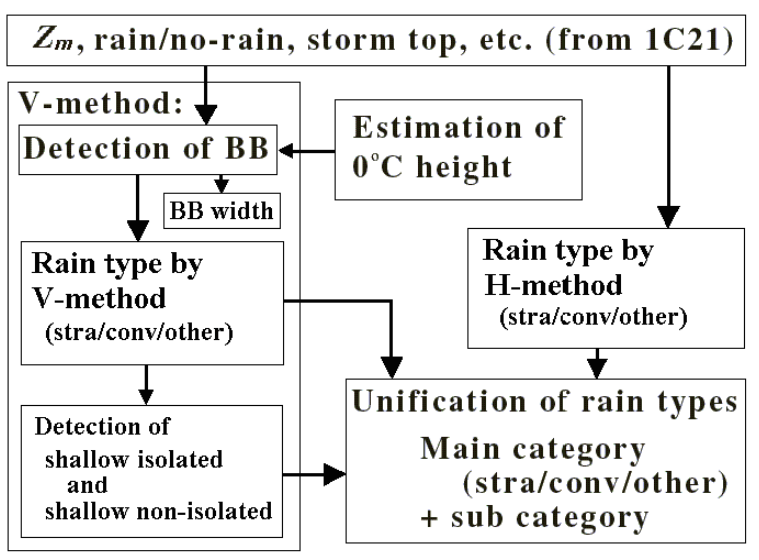

Fig. 1. Algorithm flow diagram of 2A23 V6. Rain type is classified into stratiform (stra), convective (conv), and other by two independent methods: the V-method and the H-method. The results of these are unified, and 2A23 V6 outputs the unified rain type, whose major category consists of stratiform, convective, and other. BB detection plays an important role in the V-method. 2A23 V6 also detects shallow isolated rain and shallow non-isolated rain.

of the stratiform rain is widespread because BB appears in a widespread area and because the second characterization means that stratiform rain is not convective rain, which is localized. In the above, $Z$ of the rain pixel means the maximum value of $Z$ in the rain region along the beam, that is, the maximum value of $\mathrm{Z}$ in the height region that is lower than HBB when $\mathrm{BB}$ is detected, or lower than the estimated $0^{\circ} \mathrm{C}$ height when BB is not detected, by about $1 \mathrm{~km}$.

(2) Rain type is convective when BB is not detected and when one of the following conditions is satisfied. (2a) $\mathrm{Z}$ of the rain pixel is very strong, or $\mathrm{Z}$ of the rain pixel stands out against the surrounding area (Steiner et al. 1995). (2b) Shallow isolated rain is detected. The data show that the observed $\mathrm{Z}$ of shallow isolated rain is small but well above the noise level.

(3) Rain type is other (3a) when there are clouds only or (3b) when the echo probably is not real but just noise although $1 \mathrm{C} 21$ marks it as rain certain.

In (3a) above, cloud only means that the precipitation echo exists only in a region above the estimated $0^{\circ} \mathrm{C}$ height by $1 \mathrm{~km}$ or more when the radar echo is examined along a given antenna beam.
All shallow isolated rain is convective in 2A23 V6, which is based on the discussion by Schumacher and Houze (2003), but the shallow non-isolated rain can be either stratiform or convective depending on the strength of Z. Most shallow non-isolated rain is stratiform in 2A23 V6. (Algorithm 2A23 V5 has only the shallow isolated rain category, and most of the shallow isolated rain is classified as stratiform simply because $\mathrm{Z}$ is not strong enough to be convective.)

\section{$2.2 B B$ detection in $2 A 23$}

The V-method of 2A23 detects the melting layer (i.e., bright band (BB)). The algorithm applies a spatial filter method (Awaka et al. 2007) and searches for the $\mathrm{BB}$ peak in the following BB height window.

$$
\text { [Hfreeze - 2.5, Hfreeze + 2.5] (km). }
$$

Here, Hfreeze is the estimated $0^{\circ} \mathrm{C}$ height, which is computed by

$$
\text { Hfreeze }=(\mathrm{Ts}-273.13) / 6.0,
$$

and $\mathrm{Ts}[\mathrm{K}]$ is a climatological temperature at SL. The lapse rate of the atmospheric temperature is assumed to be $6.0[\mathrm{~K} / \mathrm{km}]$. (In the previous $2 \mathrm{~A} 23 \mathrm{~V} 5, \mathrm{BB}$ was detected in the narrower $\mathrm{BB}$ height window of Hfreeze $\pm 1.5 \mathrm{~km}$. However, testing of 2A23 V5 indicated some BB outside of this height window. Hence, a wider window is used in 2A23 V6.) The climatological data of temperature at SL is a static and consists of 12 months of data. This climatological temperature data was provided by NASA just before the launch of the TRMM satellite and has not been updated since.

The $\mathrm{Z}$ profile peak is judged to belong to $\mathrm{BB}$ when it satisfies certain conditions, which means that $\mathrm{BB}$ is detected.

When $\mathrm{BB}$ is detected, the width of $\mathrm{BB}$ is calculated in 2A23 V6 by determining the upper and lower boundaries of BB. Details are given in Appendix A.

\section{Statistical results}

\subsection{BB count}

Since the TRMM PR scans the antenna perpendicular to the satellite movement, the statistics of the PR products are angle-bin dependent. Though the main topic of this Subsection is the BB count, rain type counts are also discussed because BB is closely related to rain type classification. 
Figure 2 illustrates the angle-bin dependence of the BB count. Angle bin number 25 corresponds to the nadir direction, and angle bin numbers 1 and 49 correspond to the scan edges (see Table 1). Figure 2 also presents angle-bin dependences of the stratiform count, convective count, and other count. Each count means an averaged one-month count, which is defined as the total count of each item divided by the number of months. The solid lines plot counts before the boost, and the dotted line plots those after the boost. The total data over water and over land (over water + land) were used for the analysis.

Though the figure is very congested, the following important observations on BB count are immediately obtained. (1) BB is detected in only about $40 \%$ of stratiform rain (see also Fig. 3). (2) The BB count is strongly angle-bin dependent. (3) The BB count becomes smaller after the boost.

Let us first discuss how the counts depend on the angle bin (Fig. 2). The difference between the counts before and after the boost will be discussed later. The spikes appearing in the figure will also be briefly discussed.

Figure 2 indicates that the stratiform count and the convective count depend on the angle bin, but the other count is almost independent of the angle bin. The detected BB count exhibits a large dependence on the angle bin.

The stratiform count is rather constant near the nadir but decreases near scan edges because the stratiform precipitation includes a large number of shallow non-isolated rain events. When the storm top is low, the low rain echo is masked by a smeared surface clutter, particularly near scan edges.

Figure 2 demonstrates that the convective count also depends on the angle bin. If most of the convective precipitation has a tall storm top and a strong precipitation rate, the convective count would be almost independent of the angle bin because the high storm top is free from the smearing effect of surface clutter. Nonetheless, the figure still exhibits angle bin dependence simply because all the shallow isolated rain is classified as convective in 2A23 V6. The data before the boost suggests that the convective count becomes almost independent of the angle bin if we exclude the shallow isolated count (Awaka et al. 2007). (We can arrive at a similar conclusion for the data after the boost if we subtract a slight right-left asymmetry from the data.)

The other count in Fig. 2 is almost constant over the angle bin because the other type in 2A23 V6 consists

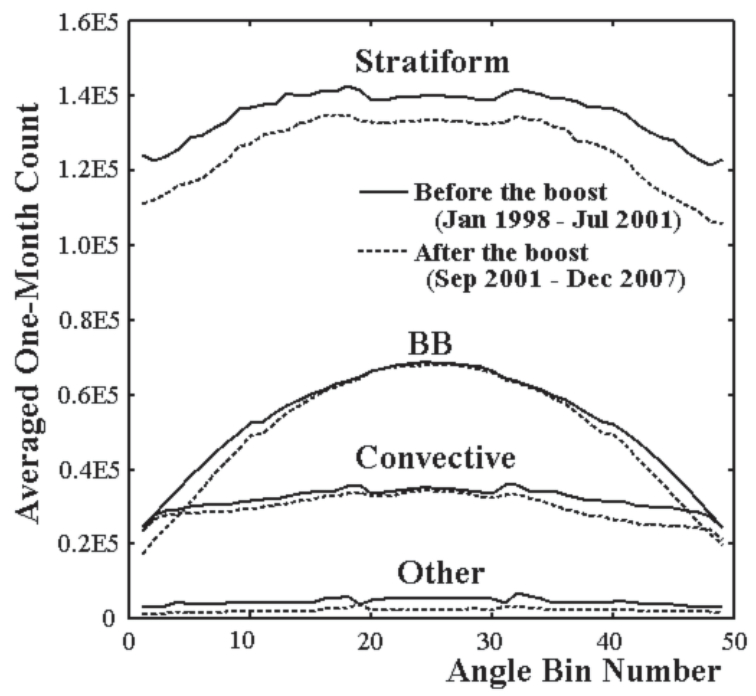

Fig. 2. Angle bin dependence of $\mathrm{BB}$ count, together with stratiform count, convective count, and other count. Each count means averaged one-month count. Ten years of all the data (over water+land) were used in the plot. Solid lines represent the data before the boost, and dotted lines, that after the boost. The BB count strongly depends on the angle bin number, which specifies the antenna scan angle. Angle bin number 25 corresponds to the nadir direction.

of only a cloud case and a noise case (see Section 2.1). In the tropics, and also outside the tropics except in winter at higher latitude in the TRMM PR observation, clouds are uniform over all angle bins in a statistical sense because of the high storm top, which is too high to be masked by the surface clutter. The noise may also appear uniformly over all angle bins because of its randomness. For these reasons, the other count becomes almost constant with respect to the angle bin.

In Fig. 2, the BB count exhibits a large dependence on the angle bin, because the smearing of the observed $\mathrm{BB}$ echo profile becomes stronger as the angle bin approaches the scan edges. The smearing effect makes the detection of BB very difficult, particularly near scan edges. The mechanism of the smearing of BB echo is essentially the same as that of the surface echo (which is a clutter for the rain observation).

Next, let us discuss the difference between the 
counts before the boost and those after the boost.

Figure 2 clearly demonstrates that each averaged one-month count became smaller after the boost. The figure also indicates that all the counts decrease more rapidly after the boost when the angle bin approaches the scan edges.

These features can be easily understood because the altitude change has the following impacts on the data. (I1) The boost slightly decreased the sensitivity of PR because of the increase in distance between PR and the target. In terms of $\mathrm{Z}$, the decreased sensitivity increases the threshold level of the detectable $\mathrm{Z}$ and makes low $\mathrm{Z}$ values undetectable.

(Note that the decrease of sensitivity does not change $\mathrm{Z}$ because the radar equation (e.g., Battan 1973; Meneghini and Kozu 1990; Bringi and Chandrasekar 2001) accounts for the decreased received power.)

(I2) The boost increased the scattering volume size, which would slightly decrease measured $\mathrm{Z}$ because of the change in non-uniform filling (NUF) of the precipitation within the scattering volume.

The boost increased the local zenith angle of the antenna beam because of the curvature of the Earth. The increase in the local zenith angle has the following two impacts on the data.

(I3a) The surface echo becomes more smeared, which decreases the probability of observing shallow rain, particularly near the scan edges.

(I3b) The BB peak profile becomes more smeared, which makes the detection of BB difficult, particularly near the scan edges.

The boost increased the footprint size of the radar beam. The increased footprint size has the following impacts on the data.

(I4a) Impact I3a is enhanced.

(I4b) Impact I3b is enhanced.

The increased footprint size also causes overlapping of footprints in the satellite travel direction. Since the satellite orbits the Earth more slowly when the altitude increases, the sampling interval on the surface along the track changed from $4.3 \mathrm{~km}$ before the boost to $4.25 \mathrm{~km}$ after the boost, which is only about a $1 \%$ change. Hence, the PR observation continues with the increased footprint size and with almost the same $4.3 \mathrm{~km}$ sampling interval on the surface after the boost, causing the footprints to overlap along the satellite track. The overlap of footprints means the overlap of scattering volumes of adjacent beams at the same height. Since the BB appears at almost the same height and since the BB appears in a widespread area, the overlap of footprints could increase the maximum value of $\mathrm{Z}$ in $\mathrm{BB}(\mathrm{ZmaxBB})$ as indicated in the following example.

Consider a case before the boost in which a pixel having a small ZmaxBB is located between pixels having larger $\mathrm{ZmaxBB}$ along the satellite track. If the same BB were observed after the boost, the small ZmaxBB would be increased because of the overlap of footprints. Of course, we can easily think of other examples where the overlap of footprints makes ZmaxBB smaller after the boost. It can be demonstrated, however, that the overlap of footprints can produce a large increase of $\mathrm{ZmaxBB}$ in $\mathrm{dB}$ value, but the decrease of $\mathrm{ZmaxBB}$ is small. This means that the overlap of footprints can increase ZmaxBB in a statistical sense.

The effect of the overlap of footprints and its consequence can be added to the impacts of the boost.

(I5) Overlap of footprints along the satellite track increases ZmaxBB in a statistical sense.

Figure 2 indicates that the counts became more angle-bin dependent after the boost, but the figure also indicates that the nadir direction is special. The decreased stratiform count at nadir after the boost can be explained by impacts I1 and I2. The decreases of other counts at nadir after the boost can also be explained by these impacts. However, the BB count at nadir is almost the same before and after the boost. The convective count at nadir is also almost the same before and after the boost.

For BB, impact I1 does not contribute to the BB detection because, unlike the received power, $\mathrm{Z}$ is the same before and after the boost. (BB produces a large $\mathrm{Z}$, which is a physical quantity. For a given $\mathrm{Z}$, the received power changes depending on the distance between the radar and the BB of interest, but the radar equation accounts for distance changes, so the same $\mathrm{Z}$ value is retrieved.) Since the BB is detected by examining the shape of the BB peak, the increased footprint size, which causes impacts I2 and I5, can change the shape of $\mathrm{BB}$ at nadir after the boost because of the nonuniformity of $\mathrm{Z}$ in the scattering volume. However, since BB is widespread and is very uniform, the change in the shape of BB peak at nadir after the boost is expected to be small.

Though the BB peak shape may change, the BB peak shape at nadir remains very clear and detectable after the boost. Because of this, the BB count at nadir is almost the same before and after the boost (Fig. 2).

Let us next discuss the convective count at nadir. As described in Section 2.1, algorithm 2A23 V6 
classifies the rain type as convective in the following two cases: (a) when $Z$ of the rain pixel is very strong or when $\mathrm{Z}$ of the rain pixel stands out against the surrounding area, and (b) when rain is classified as shallow isolated. Note that of the boost impacts, only impacts $\mathrm{I} 1$ and $\mathrm{I} 2$ can affect convective rain at nadir. However, the impact I1 does not affect the convective rain because, in both cases (a) and (b), $\mathrm{Z}$ is well above the noise level. (As mentioned in Section 2.1, the data indicate that the observed $\mathrm{Z}$ of shallow isolated rain is small but is well above the noise level.) Therefore, only impact I 2 affects convective rain at nadir.

In the convective rain case (a), impact I2 would cause some $\mathrm{Z}$ of the rain pixel to become so small that $\mathrm{Z}$ of the rain pixel does not satisfy the very strong condition, but such a case is covered by the stand-out condition. The stand-out condition would not be affected by impact I 2 because the impact affects both the $\mathrm{Z}$ of interest and those in the surrounding area. Thus, impact $I 2$ does not change the convective count for case (a) at nadir.

The count at nadir for case (b) of convective rain, that is, for the case of shallow isolated rain, can be explained as follows. Impact $\mathrm{I} 2$ causes a smaller $\mathrm{Z}$ after the boost, but the impact does not change the isolated condition. A small decrease of $\mathrm{Z}$ of the shallow isolated rain at nadir would not drive $\mathrm{Z}$ below the noise level. Hence, the shallow isolated count at nadir, that is, the convective count for case (b) at nadir, would not change appreciably before and after the boost.

Since the convective counts at nadir for case (a) and case (b) do not change appreciably before and after the boost, the convective count at nadir is almost the same before and after the boost (Fig. 2).

There is another impact of the boost on the data, however. Figure 2 reveals a slight right-left asymmetry after the boost. This happened because of a beam mismatch at the switching instant of the antenna beam direction (Takahashi and Iguchi 2004). This beam mismatch did not exist before the boost because PR was designed to work perfectly at an altitude of 350 $\mathrm{km}$.

The last subject in Fig. 2 is the spikes in rain type counts and also on a small discontinuities in the BB count. The spikes appear in the rain type counts of Fig. 2 around angle bin numbers 18 and 32. These spikes appear because of side-lobe clutter (Kozu and Meneghini 1990), which appear very close to the surface echo and are misjudged as rain echoes. When the side-lobe clutter appears in the shape of a clear peak above the surface echo, it is easy to reject the side- lobe clutter by a side-lobe rejection filter, but when the side-lobe clutter appears very close to or adjacent to the surface echo, the side-lobe rejection filter does not work well, resulting in the appearance of spikes in the rain type counts. Since BB is usually detected at heights well above the surface echo, the side-lobe clutter does not affect the BB detection, and in fact the $\mathrm{BB}$ count is smooth around angle bin numbers 18 and 32 .

The BB count, however, exhibits small discontinuities around angle bin numbers 11 and 39 due to changes in the parameters for detecting BB at angle bin numbers 11 and 39 to accommodate smearing of BB. It would be preferable to lessen the discontinuity of BB count in the next version of $2 \mathrm{~A} 23$.

Table 2 lists the percentage of each rain type count before and after the boost.

The changes of percentage are small but discernible. After the boost, the percentage of the stratiform count against the total rain count increased slightly, the percentage of the convective count remained almost the same, and the percentage of the other count decreased appreciably. Table 2 also presents the contribution of the data over land and that over water.

Figure 3 shows the percentage of the ratio of $\mathrm{BB}$ count to stratiform count. This information is important because not all the stratiform rain is associated with the detected BB in 2A23. The thick solid line in Fig. 3 represents the case of using all the stratiform data (over water+land); the thin solid line, the case of using the data over water only; and the dotted line, the case of using the data over land only.

Figure 3 also indicates that the percentage of the ratio of $\mathrm{BB}$ count to stratiform count for all the data (over water+land) slightly increased after the boost (the average ratio for all data (over water+land) was $39.3 \%$ before the boost and $40.1 \%$ after the boost), though the BB count itself decreased after the boost (Fig. 2). This occurred because the percentage of the ratio of $\mathrm{BB}$ count to stratiform count in Fig. 3 is a conditional percentage. As indicated in Fig. 2, both the BB count and the stratiform count decreased after the boost, but it is easy to see in Fig. 2 that the stratiform count decreased more after the boost than the $\mathrm{BB}$ count, which slightly increases the ratio of $\mathrm{BB}$ count to stratiform count after the boost.

Figure 3 demonstrates that the ratio of $\mathrm{BB}$ count to stratiform count over water and that over land are almost the same on average, but the fluctuations are much larger in the data over land. The standard deviation of the ratio over water was 0.79 before the boost 
Table 2. Percentages of rain type counts before and after the satellite boost.

\begin{tabular}{l|c|c}
\hline & $\begin{array}{c}\text { Before the boost } \\
\text { Jan 1998 - Jul 2001 }\end{array}$ & $\begin{array}{c}\text { After the boost } \\
\text { Sep 2001 - Dec 2007 }\end{array}$ \\
\hline Stratiform & 78.6 & 79.6 \\
(over water) & $(59.6)$ & $(61.2)$ \\
(over land) & $(18.9)$ & $(18.4)$ \\
\hline Convective & 18.7 & 18.9 \\
(over water) & $(15.1)$ & $(15.0)$ \\
(over land) & $(3.6)$ & $(3.9)$ \\
\hline Other & 2.7 & 1.5 \\
(over water) & $(1.7)$ & $(0.9)$ \\
(over land) & $(1.0)$ & $(0.6)$ \\
\hline
\end{tabular}

and 0.74 after, whereas the standard deviation of the ratio over land was 1.9 before the boost and 1.8 after. One reason for this difference between the fluctuation of the ratio over water and over land is that the $\mathrm{BB}$ count over land consists of only $25 \%$ of the total BB count (as can be shown), but this percentage alone

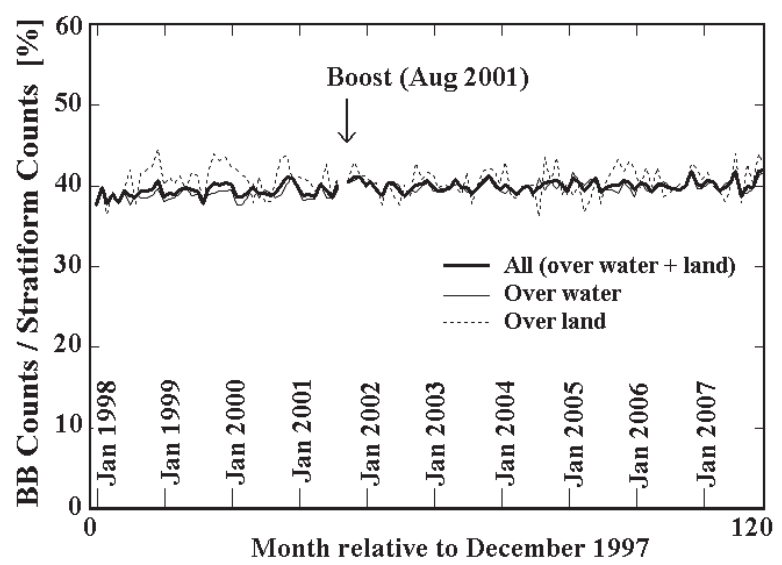

Fig. 3. Time series of the percentage of the ratio of one month $\mathrm{BB}$ count to one month stratiform count. The thick sold line plots the ratio using all the data (over water+land); the thin solid line, the ratio using the data over water; and the dotted line, the ratio using the data over land. The ratio for the data over water is very close to the ratio for all the data. The ratio for the data over land exhibits a large monthly variation. does not seem to explain the difference between the fluctuations of the ratio over land and over water. The large fluctuation of the ratio of $\mathrm{BB}$ count to the stratiform count over land seems to reflect some real phenomenon.

\section{2 $\mathrm{BB}$ height and $0^{\circ} \mathrm{C}$ height}

One of the greatest questions about BB detection by 2A23 is the use of climatological temperature data for estimating $0^{\circ} \mathrm{C}$ height in the initial part of $\mathrm{BB}$ detection (Fig. 1). This question can be answered by comparing the detected height of $\mathrm{BB}(\mathrm{HBB})$ with the $0^{\circ} \mathrm{C}$ height calculated from analysis data. This paper uses NCEP2 four-times daily data for the analysis data available at 2400 UT (Universal Time), 0600 UT, 1200 UT and 1800 UT.

Figure 4 presents scatterplots of detected $\mathrm{HBB}$ versus the $0^{\circ} \mathrm{C}$ height calculated by using NCEP2 (HfreezeNCEP2). In each figure, a line with a $45^{\circ}$ inclination is drawn in order to facilitate comparing HBB and HfreezeNCEP2.

HfreezeNCEP2 was calculated in two steps. First, HfreezeNCEP2 was calculated by a linear interpolation at the exact grid point using air temperature data at 17 pressure levels and geopotential height data at 17 pressure levels; the grid has a spatial resolution of $2.5 \times 2.5$ degrees. Since NCEP2 data are limited to those at 2400 UT, 0600 UT, 1200 UT and 1800 UT, the above HfreezeNCEP2 was calculated at two successive two UTs straddling the BB observation time. Second, HfreezeNCEP2 at the observation point by the PR was tempo-spatially interpolated. A linear interpolation was used for the temporal interpolation, and a bilinear interpolation was used for the spatial interpolation.

The scatterplot in Fig. 4 is actually a plot of twodimensional histogram of the percentage of the BB count; the interval of the two-dimensional histogram is $0.02 \mathrm{~km} \times 0.02 \mathrm{~km}$.

Figure 4 presents a set of scatterplots acquired in February 1998; the data of this month have been used for benchmark testing of the TRMM PR algorithms. Figure $4 \mathrm{a}$ depicts the scatterplot for all the data (over water+land); Fig. 4b, the scatterplot for the data over water; and Fig. 4c, the scatterplot for the data over land. These figures use all the latitude and all the longitude data. However, Fig. 4 gives us some information about the dependency of the scatter of HBB versus HfreezeNCEP2 on the latitude because HBB and HfreezeNCEP2 are expected to be higher in the tropics. In Fig. 4, HfreezeNCEP2 would be reliable. 
Hence, the right-hand side of each panel in Fig. 4 primarily indicates the scatter of the data in the tropics and of summer data in the southern hemisphere, and the left-hand side, the scatter of winter data in highlatitude regions of the northern hemisphere.

The height of the detected BB can deviate greatly from HfreezeNCEP2 since 2A23 detects BB within a $\mathrm{BB}$ window based on the climatological temperature data at SL (see Section 2.2). In fact, Figs. 4a through $\mathrm{c}$ indicate that some detected HBB are differ significantly from HfreezeNCEP2, but most of the significantly deviating data, which we call outliers, compose less than $1 \%$ of the data, suggesting that most of these outliers are false BBs misjudged as BBs by $2 \mathrm{~A} 23 \mathrm{~V} 6$.

In most cases, a false BB appears when 2A23 V6 misjudges a peak in the height profile of convective rain as the true peak of $\mathrm{BB}$. A typical height profile of $\mathrm{Z}$ for convective rain measured by the TRMM PR is as follows. When the measured $\mathrm{Z}$ is examined from high to low altitudes, the measured $\mathrm{Z}$ begins to increase suddenly at a certain height and reaches to the height of a maximum value of $Z$. After this height, the measured $\mathrm{Z}$ decreases with decreasing height because of the strong attenuation of convective rain. In this way, a peak appears in the height profile of convective Z. Usually, such a peak of convective rain is broadened and is not detected as a BB peak. Sometimes, however, it becomes indistinguishable from the true $\mathrm{BB}$ peak and is detected as a BB peak by 2A23 V6. This is one type of false BB.

Another type of false BB, although not so frequent, occurs when shallow isolated rain is misjudged as BB because Z, when examined from high to low altitudes, may suddenly increase at the top of shallow isolated rain. The measured $\mathrm{Z}$ reaches a maximum and then decreases slightly in the next range bin and subsequently remaining almost constant in height, thus making a small peak at the height of maximum Z. Algorithm 2A23 sometimes misjudges this small peak as a BB peak.

Event data analysis (not shown) indicates that the outliers tend to appear when few BB are detected in one antenna scan of data, consisting of 49 angle bins.

Based on this information, we reanalyzed the data by applying the following very simple filter.

Simple filter When five or fewer BBs are detected in one antenna scan, these BBs are judged as false and are filtered out in the analysis.

The lower panels of Fig. 4 present scatterplots of HBB versus HfreezeNCEP2 after the above filter is applied. Figure $4 \mathrm{~d}$ depicts the scatterplot for all the data (over water+land); Fig. 4e, that for the data over water; and Fig. 4f, that for the data over land. Outliers, in particular those outliers in the lower area of $90 \%$ to $99 \%$ levels on the right-hand part of each plot, are greatly reduced in the lower panels of Fig. 4, which demonstrates the effectiveness of the filter.

It should be noted, however, that the above filter is too simplistic. If there are only false BBs originating from strongly attenuated convective rain or from shallow isolated rain in one antenna scan of data, these false BBs would be few and would appear randomly in the angle bins of one antenna scan. However, since BBs appear in widespread stratiform rain, BB is usually detected continuously over several bins. Therefore, just a few detected BBs in one antenna scan does not necessarily mean that the detected BBs are false. If the detected BBs appear continuously in at least three consecutive angle bins, it would be highly probable that they are true BBs.

The above filter considers only the number of BBs; it does not consider the random appearance of BBs. This means that the above filter will reject both false BBs and true BBs when just a few BBs are detected in one antenna scan. A simple filter that just examines the number of detected BB would be adequate to examine the source of the outliers shown in Fig. 4, because most of the true BBs when just a few BBs are detected should appear in the main data, not as outliers. To avoid rejecting true BBs, however, several refinements to the filter are needed. Possible refinements would be realized by adding the filter a function of examining the random appearance of BB, and by adding another function of examining the number of BB not only in the direction of antenna scan but also in the direction of satellite movement.

Figure 4 indicates that the majority of HBBs are close to HfreezeNCEP2. On average, HBB appears about $0.5 \mathrm{~km}$ below HfreezeNCEP2, which is reasonable because solid-phase particles aloft (i.e., snow and ice) need to melt in the upper part of BB. This result is consistent with the result by Harris et al. (2000), who found that the HBB detected by the PR is lower than the NCEP-derived $0^{\circ} \mathrm{C}$ height by 300 to $900 \mathrm{~m}$.

In Fig. 4, the distribution of HBBs is almost the same for over water and for over land, although a large variation of surface temperature is expected over land. This, however, is not a surprise at all. Figure 4 simply indicates that NCEP2 works well for estimating HBB over land.

Let us next examine the effect of the boost on the 


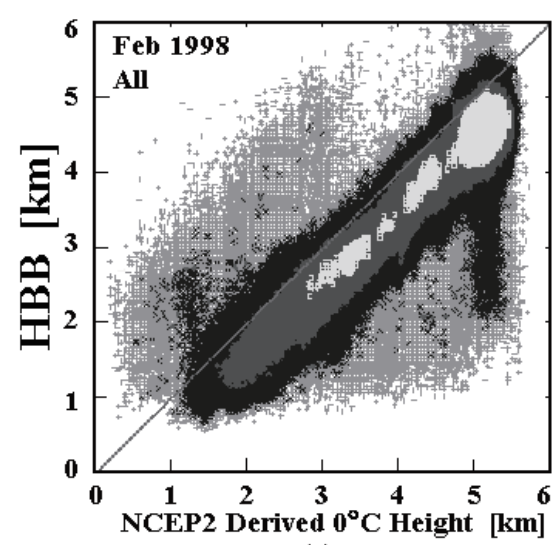

(a)

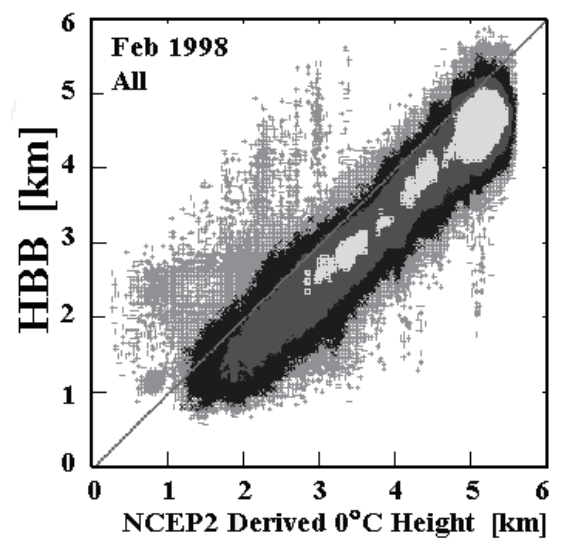

(d) With Filter

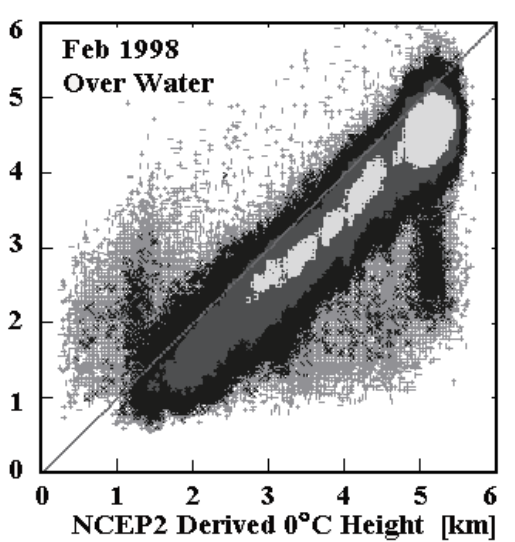

(b)

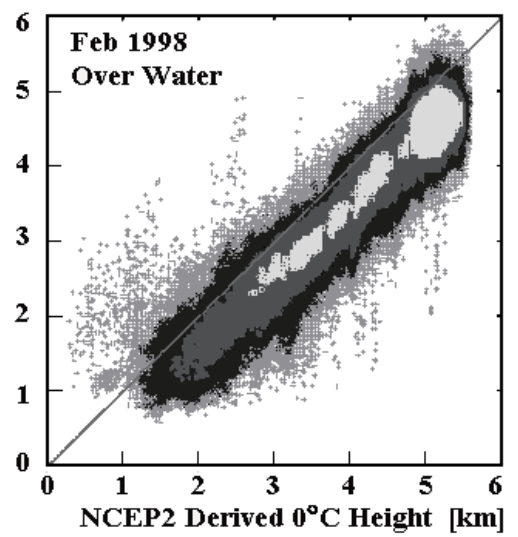

(e) With Filter

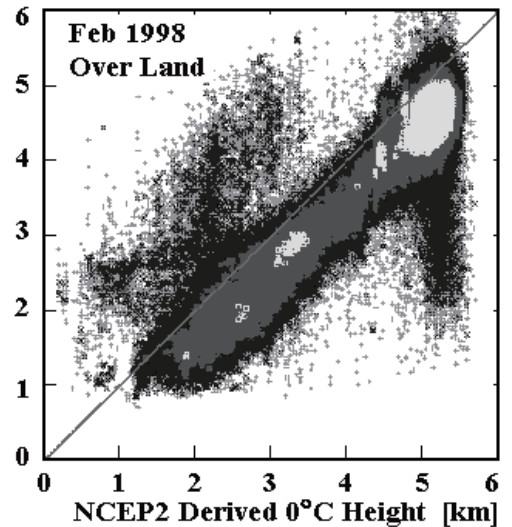

(c)

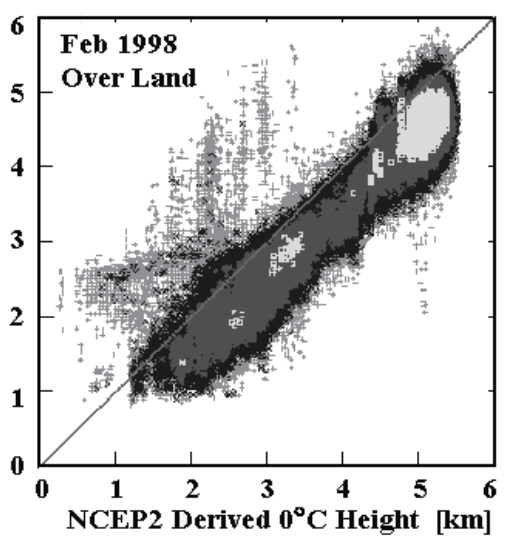

(f) With Filter

\section{$<50 \% \square 50 \%-90 \% \square 90 \%-99 \% \square 99 \%-100 \%$}

Fig. 4. Scatterplot of HBB versus NCEP2 derived $0^{\circ} \mathrm{C}$ height (HfreezeNCEP2) in February 1998. Upper panels depict the original case, and lower panels, the case after applying the simple filter explained in the text. Panels (a) and (d) present scatterplots using all the data (over water+land); panels (b) and (e), the scatterplots using the data over water; and panels (c) and (f), the scatterplots using the data over land. Data points were prepared in the form of a two-dimensional histogram with a grid interval of $0.02 \mathrm{~km} \times 0.02 \mathrm{~km}$.

relation between HBB and HfreezeNCEP2. To examine the effect of the boost, a longer-term statistic is preferable for reducing the effect of fluctuations. One year data for each are used in Fig. 5, that is, one year data from February 1998 to January 1999 before the boost and one year data from February 2002 to January 2003 after the boost.

Figure 5 presents scatterplots of $\mathrm{HBB}$ versus HfreezeNCEP2 before and after the boost; for simplicity, the figure shows only the case of using all the data (over water+land). Each panel of Fig. 5 presents a two-dimensional histogram of the percentage of the
BB count. In each panel, a line with a $45^{\circ}$ inclination is drawn in order to facilitate comparing $\mathrm{HBB}$ and HfreezeNCEP2.

In the one-year scatterplot of Fig. 5, the outliers in the percentage range of $99 \%$ to $100 \%$ are distributed in a wider area than in Fig. 4, which shows a one-month scatterplot. This occurs because the data points are additive so that the data-occupied area in the scatterplot only increases as the size of the dataset increases. We should focus our attention on the shape of the scatterplot for percentages below $99 \%$.

The upper left panel, Fig. 5a1, presents the scatter- 


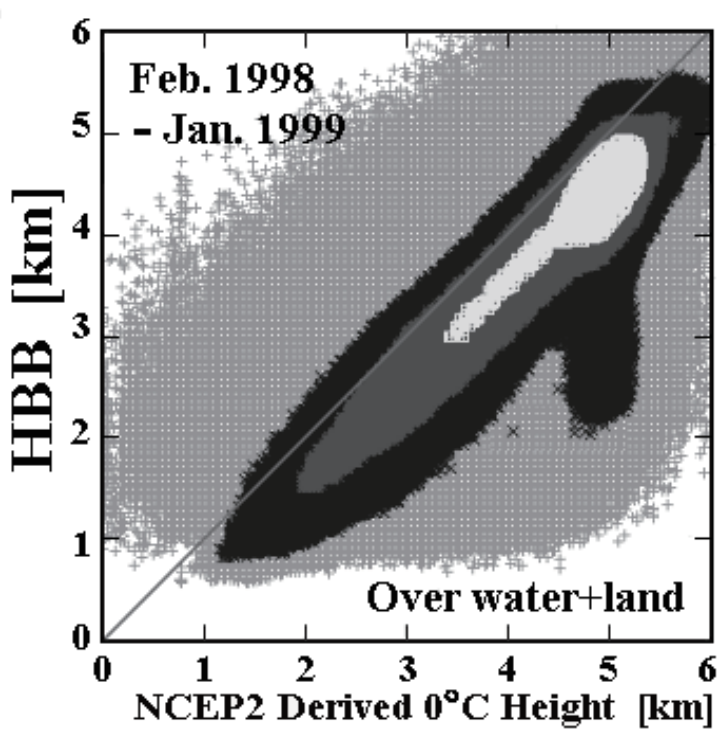

(a1) A 1-year data before the boost without the filter

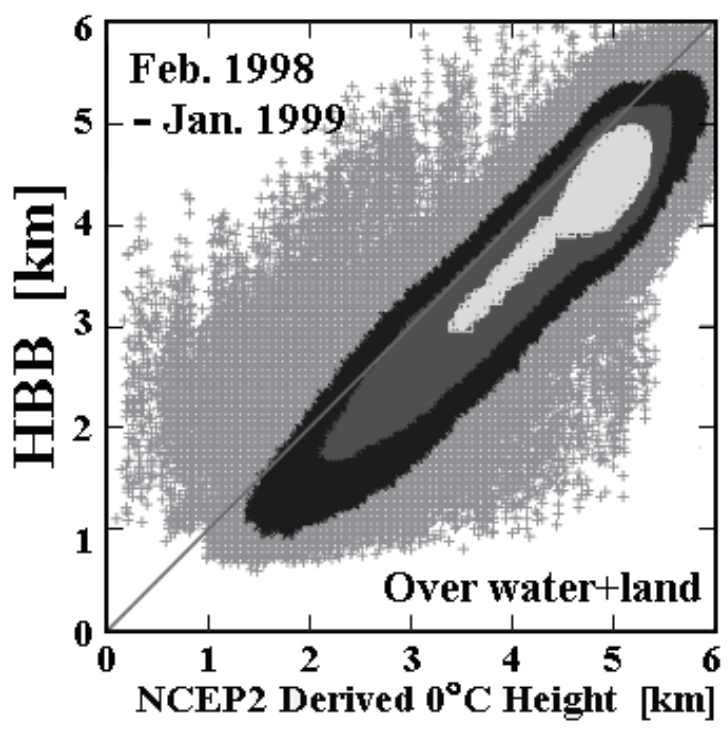

(a2) A 1-year data before the boost with the filter

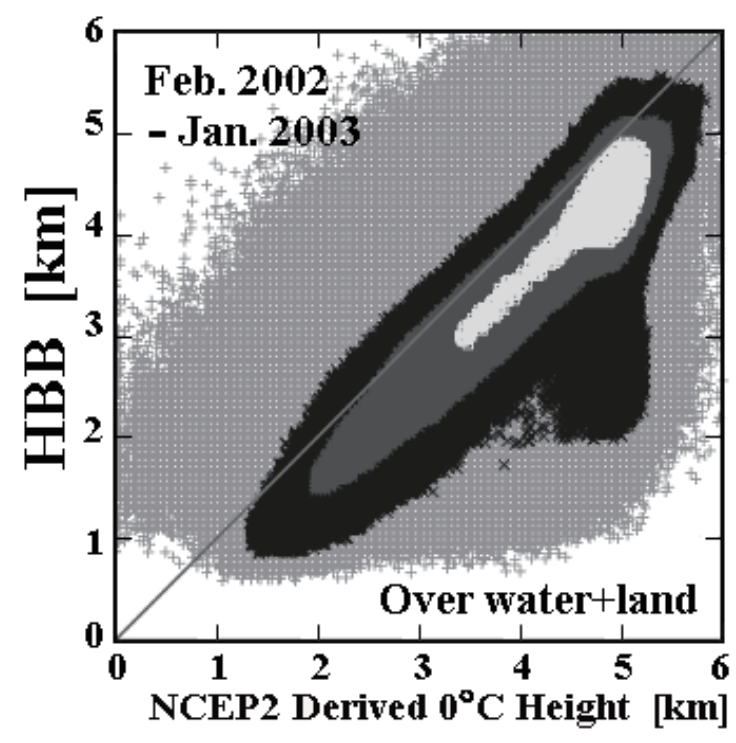

(b1) A 1-year data after the boost without the filter

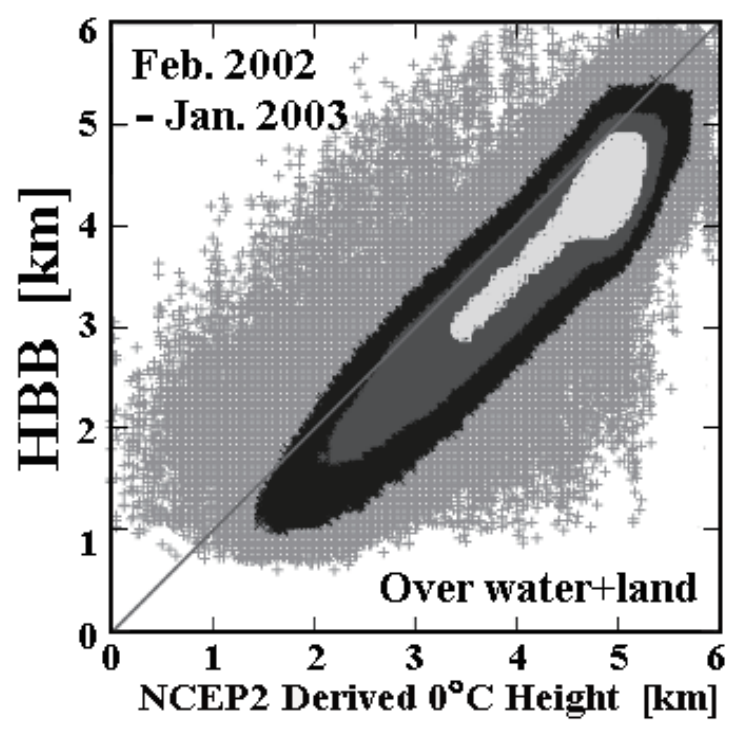

(b2) A 1-year data after the boost with the filter

$90 \%-99 \%$

$99 \%-100 \%$

Fig. 5. Scatterplot of HBB versus NCEP 2 derived $0^{\circ} \mathrm{C}$ height (HfreezeNCEP2) before and after the boost. The left panels show scatterplots using one-year data before the boost (Feb. 1998 to Jan. 1999), and the right panels, scatterplots using one-year data after the boost (Feb. 2002 to Jan. 2003). The upper panels present the original case, and the lower panels, the case after applying the simple filter explained in the text. All the data (over water+land) were used in the plot. Data points were prepared in the form of a two-dimensional histogram with a grid interval of $0.02 \mathrm{~km} \times 0.02 \mathrm{~km}$. 
plot before the boost, and the upper right panel, Fig. 5b1, shows the scatterplot after the boost. In Fig. 5b1, the data in the 90 to $99 \%$ levels indicate a slightly widened downward shape on the right-hand of the plot, primarily due to the false BBs originating from shallow isolated rain. After the boost, the shape of the $\mathrm{Z}$ profile smears more, resulting in an appreciable decrease in detected BB count. However, the false BB count, which originated from shallow isolated rain, is not so strongly affected by the boost because most shallow isolated rain is observed near the nadir direction, where the effect of smearing is small. Therefore, the percentage of the false $\mathrm{BB}$ count increases with respect to the total $\mathrm{BB}$ count in the area of interest after the boost.

The lower panels of Fig. 5 present scatterplots of HBB versus HfreezeNCEP2 when the simple filter is applied. The lower left panel, Fig. 5a2, depicts the case before the boost, and the lower right panel, Fig. $5 \mathrm{~b} 2$, that after the boost. Because of the filter, the downward shaped region for the 90 to $99 \%$ level on the right-hand side in Fig. 5a1 and that in Fig. 5b1 disappear (Figs. 5a2 and 5b2). Thus, Fig. 5 demonstrates that the simple filter very effectively filters BB out outliers both before and after the boost.

Note that the simple filter mentioned in this paper was applied to the output of 2A23 V6. The code of 2A23 was not changed in this test. However, we are planning to introduce a similar but more refined filter in 2A23 V7. Introduction of analysis data (such as Global analysis data by the Japan Meteorological Agency (GANAL), NCEP, and NCEP2) for estimating the $0^{\circ} \mathrm{C}$ height is also a possibility in $2 \mathrm{~A} 23 \mathrm{~V} 7$. If reliable analysis data is used in 2A23 V7, a much narrower BB height window can be used. We are studying the possibility of using GANAL in 2A23 V7. Use of such analysis data would be a must for the data analysis of the planned GPM DPR (Kobayashi and Iguchi 2003).

\subsection{BB statistics using $3 A 25$}

BBs can also be statistically analyzed using the level 3 TRMM PR algorithm 3A25 (Okamoto et al. 1998). In 3A25, various monthly statistics of the level 2 PR products are calculated with a standard space resolution and a high space resolution. The standard space resolution data are stored in $5^{\circ} \times 5^{\circ}$ latitudelongitude grid cells; the high space resolution data are stored in $0.5^{\circ} \times 0.5^{\circ}$ latitude-longitude grid cells.

Unless otherwise stated, the BB statistics in this subsection were obtained by using the high space resolution data of 3A25 V6.

Figure 6 presents the zonal mean of $\mathrm{ZmaxBB}$. Figure $6 \mathrm{a}$ depicts the zonal mean of ZmaxBB for all the data (over water+land); Fig. 6b, the zonal mean of ZmaxBB over water; and Fig. 6c, the zonal mean of $\mathrm{ZmaxBB}$ over land. The thick line indicates the zonal mean of ZmaxBB before the boost (January 1998 to July 2001), and the dotted line, the zonal mean of ZmaxBB after the boost (September 2001 to

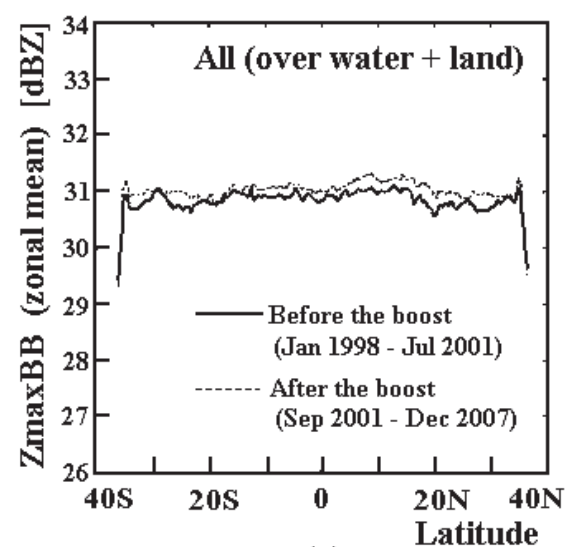

(a)

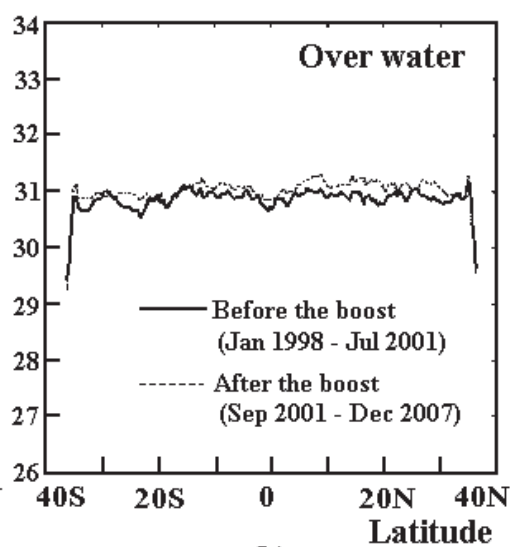

(b)

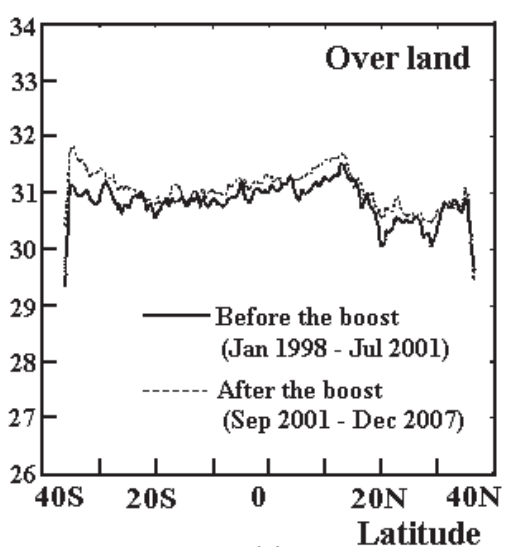

(c)

Fig. 6. Zonal mean of ZmaxBB. Abscissa of each panel represents latitude. Panel (a) shows the zonal mean of ZmaxBB using all the data (over water+land); panel (b), the zonal mean using the data over water; and panel (c) the zonal mean using the data over land. High resolution $\left(0.5^{\circ} \times 0.5^{\circ}\right.$ cells $)$ data of $3 \mathrm{~A} 25 \mathrm{~V} 6$ were used. 
December 2007). It should be noted that BB statistics in $3 \mathrm{~A} 25 \mathrm{~V} 6$ are the statistics on the BB outputs of $2 \mathrm{~A} 23 \mathrm{V6}$, which means that $\mathrm{ZmaxBB}$ in $3 \mathrm{~A} 25 \mathrm{~V} 6$ is not attenuation corrected. A small attenuation due to $\mathrm{BB}$ itself is expected in $\mathrm{ZmaxBB}$.

Figure 6 indicates that the zonal mean of $\mathrm{ZmaxBB}$ after the boost is slightly greater than that before the boost (about $0.3 \mathrm{~dB}$ ). This occurred because the zonal mean of ZmaxBB was calculated using the conditional probability (see Appendix B and Fig. 10).

From Fig. 6a, the zonal mean of ZmaxBB for all the data (over water+land) is about $31 \mathrm{dBZ}$, which is almost constant in latitude (except at the northern and southern latitude boundaries). A similar observation can be made in Fig. 6b, e.g., the zonal mean of ZmaxBB over water is about $31 \mathrm{dBZ}$ and is almost constant in latitude (except at northern and southern latitude boundaries). The above observations suggest that the BB properties are similar in latitude at least in the over water case. However, Fig. $6 c$ shows that the zonal mean of ZmaxBB over land is slightly smaller in regions above 20 degrees latitude in the northern hemisphere. Before the boost, the zonal mean of ZmaxBB over land exhibited two clear dips, one at about a latitude of $20^{\circ} \mathrm{N}$, and the other at about $30^{\circ} \mathrm{N}$. After the boost, two similar dips seem to exist but they are not so clear. The appearance of these two dips in the zonal mean of ZmaxBB over land seems to be related to the fact that the dry area of North Africa is located at about $20^{\circ} \mathrm{N}$ latitude, and the Himalaya
Range is located at about $30^{\circ} \mathrm{N}$ latitude (for a global TRMM rainfall map, see Liu et al. 2008).

Figure 6 indicates a sudden decrease of $\mathrm{ZmaxBB}$ at both the northern and southern latitude boundaries (of the observable area). There is no such strange phenomena when the normal space resolution $\left(5^{\circ} \times\right.$ $5^{\circ}$ grid cells) data of $3 \mathrm{~A} 25 \mathrm{~V} 6$ are used (not shown), which may imply that the strange behavior had occurred because of a smaller BB count at both north and south latitude boundaries. Because of this, we examine the BB count next.

Figure 7 illustrates the dependency of the zonal total of BB count on latitude. Figure 7a presents the zonal total of BB count of all the data (over water+land); Fig. 7b, the zonal total of $\mathrm{BB}$ count over water; and Fig. 7c, the zonal total of BB count over land. The ordinate of Fig. 7 represents the averaged one-month BB count, which is defined as the zonal total of BB counts at each latitude bin divided by the number of observation months. The ordinate of each figure is expressed on a logarithmic scale. The thick line plots the zonal total of BB count per month before the boost, and the dotted line, that after the boost.

The averaged one-month BB count near both the northern and southern latitude boundaries increased significantly due to the widely known effect of a satellite sampling (Oki and Sumi 1994) in which a satellite operating at a given inclination angle observes a certain location on the Earth more frequently as the latitude becomes higher. We should note that, although

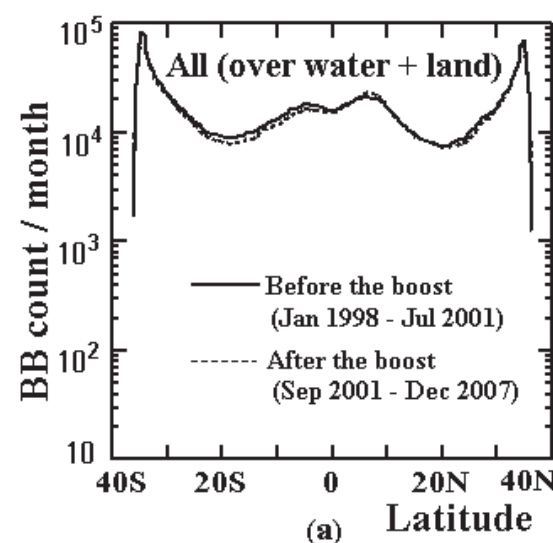

(a)

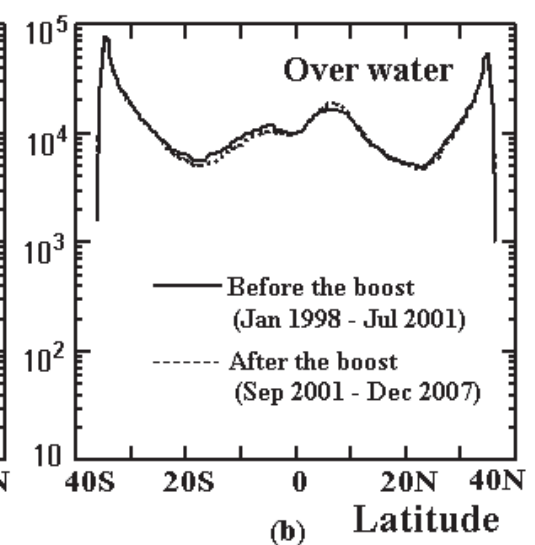

(b)

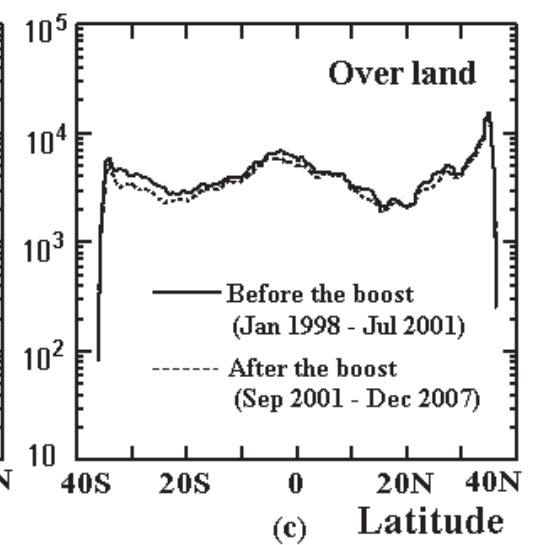

Fig. 7. Averaged one-month zonal total of BB count before and after the boost. Abscissa of each panel represents latitude. Panel (a) shows the zonal total of BB count using all the data (over water+land); panel (b), the zonal total of BB count using the data over water; and panel (c), the zonal total of BB count using the data over land. High resolution $\left(0.5^{\circ} \times 0.5^{\circ}\right.$ cells $)$ data of $3 \mathrm{~A} 25 \mathrm{~V} 6$ were used for the plot. 


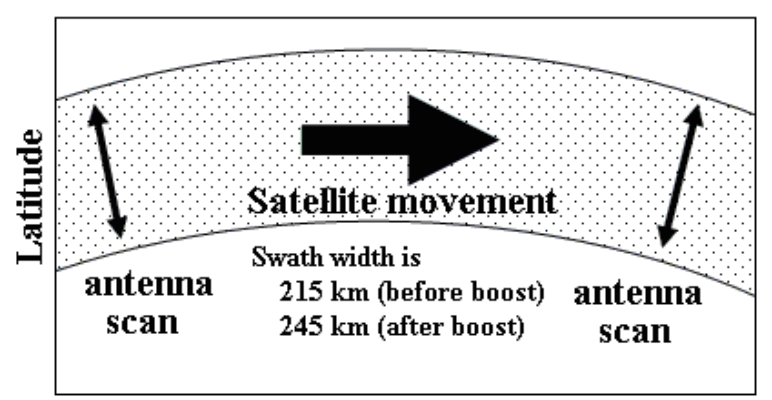

Longitude

Fig. 8. PR swath near the north latitude boundary.

there is a sharp drop of the averaged one-month BB count at the northern and southern latitude boundaries in Fig. 7, the total count during the observation period at each of these latitudes is high because there are 43 months before the boost (January 1998-July 2001) and 76 months after the boost (September 2001-December 2007). With such a large number of counts, the averages in Fig. 6 should be reliable, which means that the drops in the zonal mean of $\mathrm{ZmaxBB}$ at both latitude boundaries in Fig. 6 cannot be explained by the BB count.

Furthermore, although the BB count is indeed lower at both the northern and southern latitude boundaries, the lower BB count may not explain the fact (Fig. 6) that the changes of $\mathrm{ZmaxBB}$ at the latitude boundaries look similar and the changes always appear in the form of decrease in the magnitude of ZmaxBB. There must be a deeper reason for the strange behavior of $\mathrm{ZmaxBB}$ at the latitude boundaries.

Figure 8 illustrates the swath of PR near the northern latitude boundary, where the TRMM satellite moves almost parallel to the longitudinal direction, which means that the antenna beam scans almost parallel to the latitudinal direction. BB at the northern latitude boundary is detected only at the scan edges of the antenna beam (whether BB is detected at the edge of angle bin number 1 or at the edge of angle bin number 49 depends on the orientation of the satellite). At the scan edges, the BB profile smears more, which would make ZmaxBB smaller. (Actually, it can be demonstrated that the average of ZmaxBB decreases as the antenna scan angle increases, giving about $2 \mathrm{~dB}$ difference between the value at nadir and that at the scan edge). This mechanism would explain the sudden decrease of the zonal mean of ZmaxBB that appeared in each panel of Fig. 6 at the northern latitude boundary. The same argument can be applied to the behavior of the zonal mean of $\mathrm{ZmaxBB}$ at the southern latitude boundary.

In Fig. 7, the averaged one-month BB count is sometimes greater after the boost than that before the boost. For example, Fig. 7a indicates that the averaged one-month BB count at about $7^{\circ} \mathrm{N}$ latitude after the boost is slightly larger than that before the boost. This observation suggests that at some areas the averaged one-month BB count after the boost is slightly greater than that before the boost, although the global total of the averaged one-month BB count decreases after the boost. The next figure presents the results of a further study.

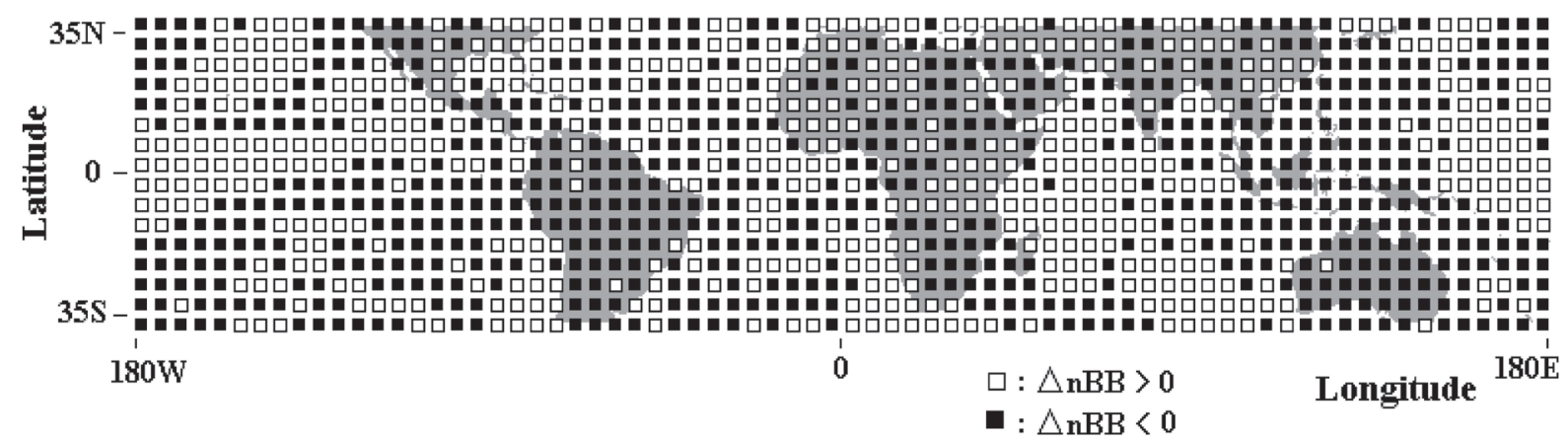

Fig. 9. Plus/minus map of $\triangle \mathrm{nBB}$ using one-year of data before the boost (January 2000 to December 2000) and after the boost (January 2002 to December 2002). A positive $\Delta$ nBB means that the averaged one-month $\mathrm{BB}$ count after the boost exceeds that before the boost; a negative $\Delta \mathrm{nBB}$ means the opposite. It is designed to be blanked out when $\triangle \mathrm{nBB}=0$, but this case does not appear in the figure. 


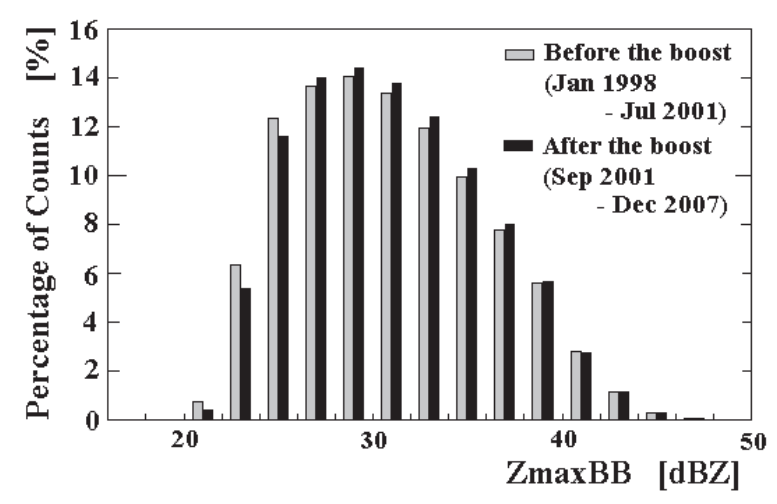

Fig. 10. Histogram of ZmaxBB. All the data (over water+land) were used for the plot. This figure was obtained by using all the angle bin data of high resolution $\left(0.5^{\circ} \times 0.5^{\circ}\right.$ cells $)$ of $3 \mathrm{~A} 25 \mathrm{~V} 6$. The gray bars indicate the histogram of $\mathrm{ZmaxBB}$ before the boost; the black bars, that after the boost. The histogram after the boost suggests an increased frequency of ZmaxBB > $26 \mathrm{dBZ}$ because of the mechanism explained at the end of Appendix B.

Figure 9 presents a plus/minus map of the difference between the averaged one-month $\mathrm{BB}$ count after the boost and that before the boost $(\triangle \mathrm{nBB})$ by using the standard space resolution $\left(0.5^{\circ} \times 0.5^{\circ}\right)$ of $3 \mathrm{~A} 25$ V6 data. In Fig. 9, one-year data acquired before the boost (January 2000 to December 2000) and One-year data after the boost (January 2002 to December 2002) were used. Figure 9 indicates that positive $\triangle \mathrm{nBB}$ occurs in many places over the globe.

Though not shown in the figure, the stratiform count and the convective count exhibit similar behavior. The increase of the BB count and the rain type counts after the boost in a certain grid region might be related to the horizontal rain structure. A further detailed study is necessary.

Figure 10 plots histograms of $\mathrm{ZmaxBB}$ for all the angle bins as the percentage of occurrence before and after the boost. All the data (over water+land) of $\mathrm{ZmaxBB}$ were used for obtaining the histograms. From Fig. 10, the percentage of smaller ZmaxBB decreases after boost, which shifts the histogram towards larger ZmaxBB after the boost (see the discussion at the end of Appendix B). The decrease of the percentage of smaller ZmaxBB after the boost can be explained as follows. After the boost, the boost im- pacts would smear the BB peak more, rendering some peaks undetectable. Note that ZmaxBB is smaller near scan edges because of the smearing, which suggests that the boost would decrease the population of smaller ZmaxBB.

Figure 11 plots the zonal mean of $\mathrm{ZmaxBB}$ at the nadir angle bin before the boost (thick line) and after the boost (dotted line). Figure 11 was obtained by plotting the ZmaxBB data of nadir BB statistics of 3A25 V6 with the standard space resolution $\left(5^{\circ} \times\right.$ $5^{\circ}$ grid cells). All the data, both over water and over land, were used for the plot. From Fig. 11, the zonal mean of $\mathrm{ZmaxBB}$ at nadir is almost constant with respect to the latitude.

Since the BB shape is sharp at the nadir angle bin, ZmaxBB in Fig. 11 is greater than that in Fig. 6a. For example, Fig. 11 indicates that the zonal mean of $\mathrm{ZmaxBB}$ at nadir before the boost is about 32 dBZ, while Fig. 6a indicates that the zonal mean of ZmaxBB before the boost is about $31 \mathrm{dBZ}$.

Figure 11 also suggests that the increase of the zonal mean of $\mathrm{ZmaxBB}$ at nadir after the boost is about

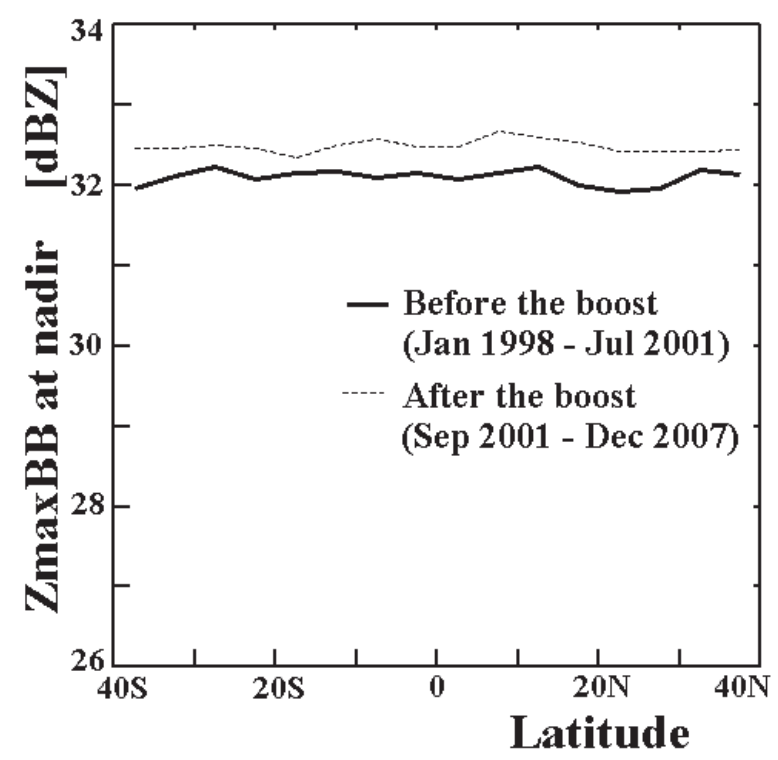

Fig. 11. Zonal mean of $\mathrm{ZmaxBB}$ at the nadir angle bin. All the data (over water+land) of the $3 \mathrm{~A} 25 \mathrm{~V} 6$ nadir BB statistics of standard resolution $\left(5^{\circ} \times\right.$ $5^{\circ}$ cells) were used for the plot. The thick solid line plots the zonal mean of ZmaxBB at nadir before the boost, and the dotted line, that after the boost. 
$0.5 \mathrm{~dB}$, which exceeds that in Fig. 6a. Histograms of ZmaxBB at nadir are necessary to understand this behavior.

Figure 12 presents histograms of $\mathrm{ZmaxBB}$ at the nadir angle bin in the form of percentage of occurrence before and after the boost. Here, the nadir BB statistics of 3A25 V6 for all the data (over water+land) with the standard space resolution $\left(5^{\circ} \times 5^{\circ}\right.$ grid cells $)$ were used. Figure 12 shows that the histogram shape shifts towards greater ZmaxBB after the boost. Comparing Fig. 10 and Fig. 12 indicates that the effect of the boost is stronger for nadir ZmaxBB in Fig. 12 than for ZmaxBB for all the angle bins in Fig. 10.

Figures 10 and 12 may look similar but the mechanisms behind them are very different. In Fig. 10, the population of smaller ZmaxBB decreases after the boost because of increased smearing. The decrease of the population of smaller ZmaxBB in Fig. 10 shifts the shape of the histogram of the percentage of $\mathrm{ZmaxBB}$ towards larger $\mathrm{ZmaxBB}$ after the boost by the mechanism explained at the end of Appendix B.

The above argument cannot be used to explain Fig. 12. Note that Fig. 12 presents the histogram of $\mathrm{ZmaxBB}$ at nadir, at which the averaged-one-month

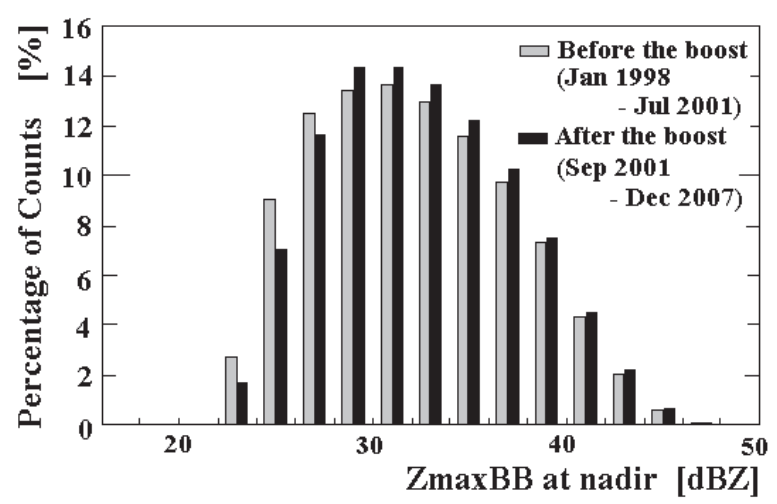

Fig. 12. Histogram of $\mathrm{ZmaxBB}$ at the nadir angle bin. All the data (both water+land) of the $3 \mathrm{~A} 25 \mathrm{~V} 6$ nadir $\mathrm{BB}$ statistics of standard resolution $\left(5^{\circ} \times 5^{\circ}\right.$ cells) were used for the plot. The gray bars plot the histogram of ZmaxBB at nadir before the boost, and the black bars, that after the boost. The histogram after the boost indicates a real increase of the count of ZmaxBB for ZmaxBB $>28 \mathrm{dBZ}$ because of impact $\mathrm{I} 5$ of the boost discussed in Section 3.1. count of BB is almost the same before and after the boost (Fig. 2). Therefore, Fig. 12 depicts a real increase of the ZmaxBB count that exceeds $28 \mathrm{dBZ}$ and a real decrease of the $Z$ maxBB count that is less than $28 \mathrm{dBZ}$. The real increase of ZmaxBB occurs because of boost impact I5 (see Section 3.1). Since overlapped footprints at nadir increases $\mathrm{ZmaxBB}$, this effect appears as greater ZmaxBB in Fig. 12. The decrease of the count of smaller ZmaxBB may be explained as follows. Some of the smaller ZmaxBBs are shifted to a greater ZmaxBB because of boost impact I5, leaving vacancies in the count of the smaller $\mathrm{ZmaxBB}$ and decreasing the BB count of smaller ZmaxBB.

\subsection{BB width}

When BB is detected, 2A23 V6 computes the width of $\mathrm{BB}$ (BBwidth), which is defined in Appendix A. The information about BBwidth would be useful for the attenuation correction in 2A25. The determination of BBwidth is rather simple and straightforward at the nadir angle bin. However, when the antenna beam moves away from the nadir direction, the BB profile smears, making it difficult to determine a reliable BBwidth, in particular near scan edges where the effect of smearing on the BB profile is very severe. Because of this, 3A25 V6 computes the statistics of BBwidth at the nadir angle bin only, though 2A23 V6 computes BBwidth at all the angle bins. Since the statistics of BBwidth are restricted to the nadir angle bin, $3 \mathrm{~A} 25$ computes only the standard space resolution $\left(5^{\circ}\right.$ $\times 5^{\circ}$ grid cells) statistics of BBwidth.

Figure 13 plots the histogram of BBwidth at the nadir angle bin before and after the boost. Oversampled data (with a $125 \mathrm{~m}$ range interval) are used to compute BBwidth in 2A23 V6, hence the BBwidth takes the value $n \times 125 \mathrm{~m}$, where $\mathrm{n}=2,3,4, \ldots$ (the minimum value of $\mathrm{n}$ is 2 otherwise we cannot detect a peak). Figure 13 indicates that the most frequently observed BBwidth is $625 \mathrm{~m}$. Figure 13 also indicates that the histogram of BBwidth is almost the same before and after the boost. A closer look at Fig. 13, however, reveals that the percentage for the $500 \mathrm{~m}$ BBwidth decreases slightly after the boost, while the percentages $750 \mathrm{~m}$ and $875 \mathrm{~m}$ BBwidths increase slightly. These observations suggest that BBwidth broadens slightly after the boost, possibly because the scattering volume increased after the boost, which slightly broadens the BB peak by an averaging effect.

The BB width increases as the BB strength increases (e.g., Klaassen 1988; Fabry and Zawadzki 1995). Therefore it would be interesting to examine the re- 


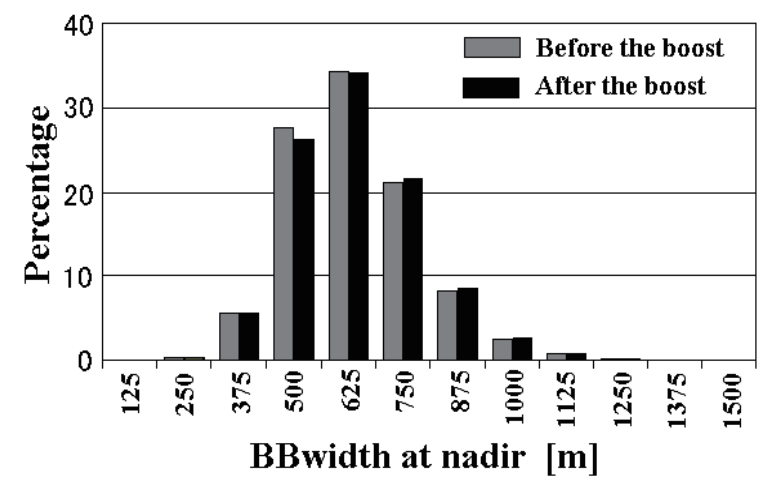

Fig. 13. Histogram of BBwidth at the nadir angle bin. All the data (over water+land) of the 3A25 V6 nadir BB statistics of standard resolution $\left(5^{\circ} \times 5^{\circ}\right.$ cells $)$ were used for the plot. The gray bars plot the histogram of BBwidth before the boost, and the black bars, that after the boost.

lation between BBwidth and $\mathrm{ZmaxBB}$, the latter of which maybe used as an indicator of BB strength. If we limit the discussion to the nadir direction, we can use the data of 3A25 V6 because 3A25 V6 computes the statistics of BBwidth and ZmaxBB at the nadir di- rection.

Let us examine the relation between BBwidth and ZmaxBB at nadir by plotting the data in a form of scatterplot using the 3A25 V6 data. (Of course, the scatterplot of BBwidth versus $\mathrm{ZmaxBB}$ at nadir can be made by using the 2A23 V6 data. Unfortunately, however, since the individual data of BBwidth at nadir takes only discrete values with a $125 \mathrm{~m}$ interval, the $2 \mathrm{~A} 23 \mathrm{~V} 6$ data is not suitable for visualizing the relation between BBwidth and ZmaxBB. As is explained in Appendix C, 3A25 V6 data is preferable for visualizing the relation between BBwidth and ZmaxBB.)

Figure 14 depicts scatterplots of BBwidth versus ZmaxBB at the nadir angle bin obtained by plotting the standard space resolution $\left(5^{\circ} \times 5^{\circ}\right.$ grid cells) data of BBwidth and ZmaxBB in 3A25 V6. All the data (over water+land) were used for the plot. Figure 14a presents the scatterplot of BBwidth versus ZmaxBB before the boost, and Fig. 14b, that after the boost.

Figure 14 demonstrates that the scatterplot before the boost (Fig. 14a) and that after the boost (Fig. 14b) have almost the same features in the sense that each figure has a similar aggregation of data points that lie in the $\mathrm{ZmaxBB}$ interval ranging from 25 to $40 \mathrm{dBZ}$ and in the BB width interval ranging from 500 to 750 m.
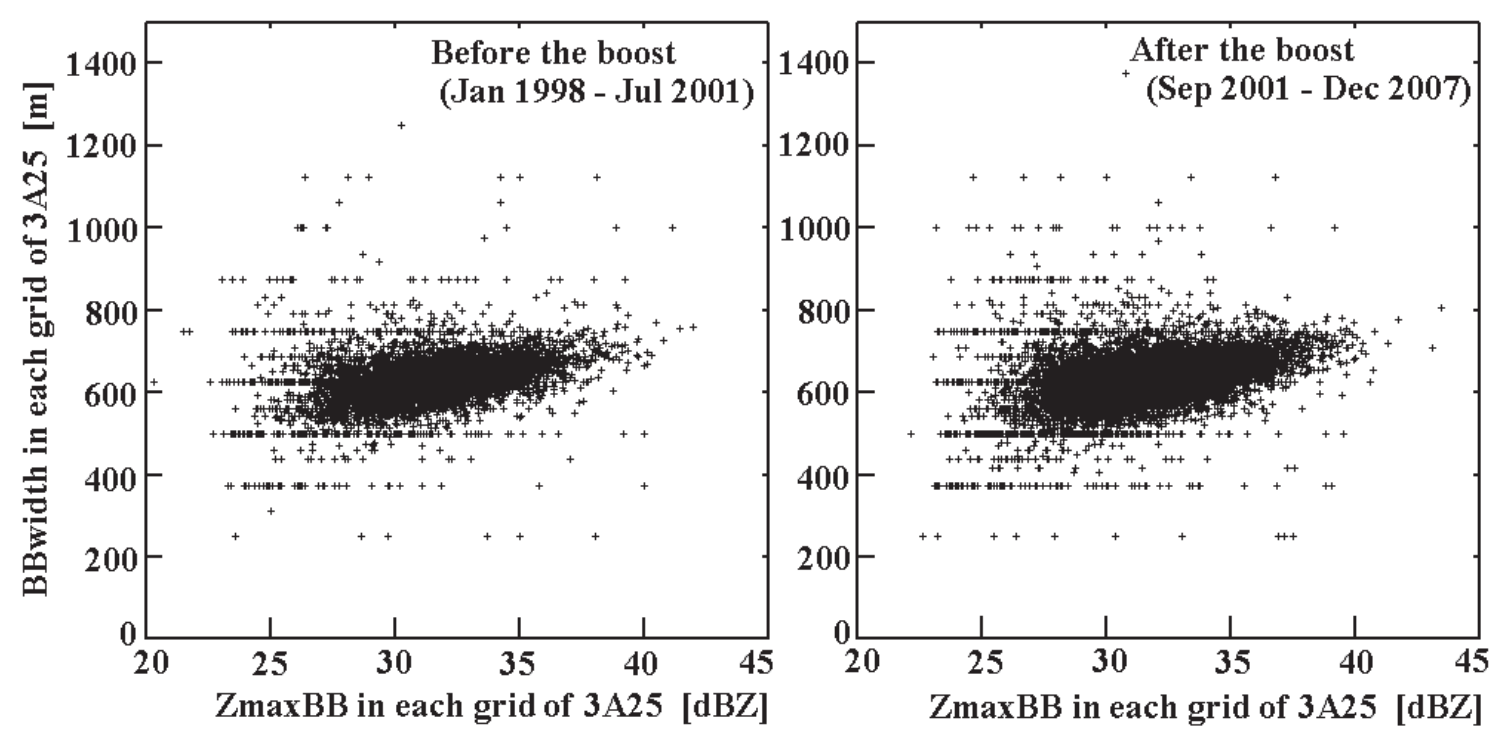

Fig. 14. Scatterplot of BBwidth versus ZmaxBB at the nadir angle bin. All the data (over water+land) of the $3 \mathrm{~A} 25 \mathrm{~V} 6$ nadir BB statistics of standard resolution $\left(5^{\circ} \times 5^{\circ}\right.$ cells) were used for the plot. The left panel shows the scatterplot before the boost, and the right panel, that after the boost. See Appendix 3 for the use of 3A25 data in the scatterplot of BBwidth versus ZmaxBB. 
Figure 14 also demonstrates that BBwidth increases as ZmaxBB increases. This result qualitatively agrees with the result reported in the literature (Klaassen 1988; Fabry and Zawadzki 1995). However, care should be taken because these authors define BBwidth differently from this paper and because they examined the relation between BBwidth and $\mathrm{Z}$ at the top of rain (Zrain). Though the definitions among the authors differ, it is the relationship between the BB width and the BB intensity, the latter of which can be characterized by different ways, that is examined. In our case, ZmaxBB is used for a measure of BB intensity because $\mathrm{ZmaxBB}$ can easily be detected.

Figure 15 plots the zonal mean of BBwidth at the nadir angle bin before the boost (thick line) and after the boost (dotted line). All the data (over water+land) were used for the plot. Figure 15 indicates that the BBwidth narrows slightly with higher latitude. This could possibly be explained as follows.

Foote and Du Toit (1969) demonstrated that the fall speed of raindrops increases with higher altitude because of lower air drag aloft. When BB exists, all the $\mathrm{BB}$ particles change into raindrops at the BB bottom. When HBB is higher, the height of BB bottom is higher, and the fall speed of raindrops at the height of $\mathrm{BB}$ bottom is higher as indicated by Foote and $\mathrm{Du}$

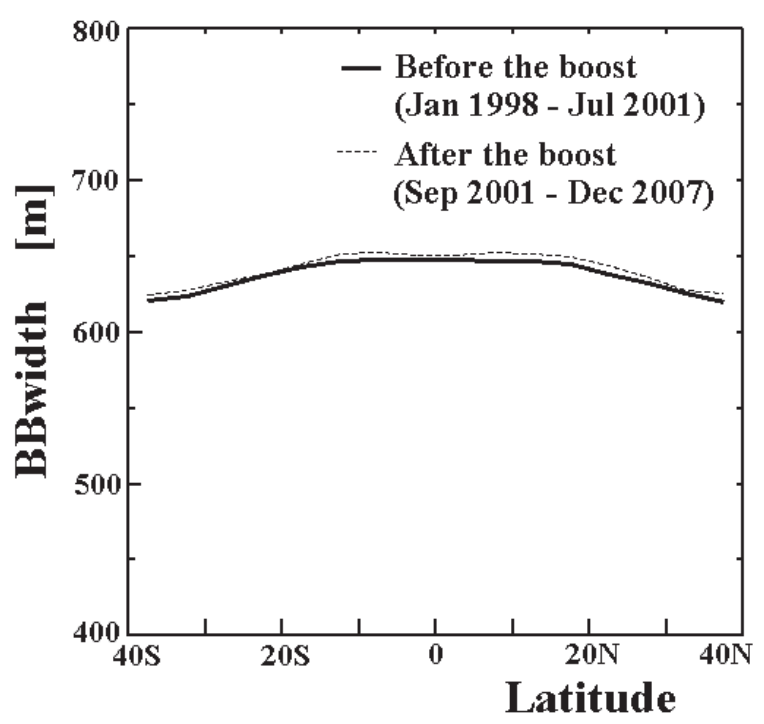

Fig. 15. Zonal mean of BBwidth at the nadir angle bin. All the data (over water+land) of the 3A25 V6 nadir BB statistics of standard resolution $\left(5^{\circ} \times 5^{\circ}\right.$ cells) were used for the plot.
Toit (1969). When the fall speed of raindrops is higher at the BB bottom, the fall speed of BB particles inside the BB would be higher. Therefore, when HBB is higher the fall speed of $\mathrm{BB}$ particles would become higher.

When the latitude becomes higher, BB appears at lower altitude, where the fall speed of BB particles would be less than that in the tropics, which means the solid particles travel a shorter distance for melting, thus reducing BBwidth.

\section{Summary}

We summarize the paper by describing its main points.

This paper first outlines TRMM PR algorithm $2 \mathrm{~A} 23 \mathrm{~V} 6$, in which $\mathrm{BB}$ is detected. Using the result of $\mathrm{BB}$ detection, 2A23 V6 classifies rain into three major categories: stratiform, convective and other.

Though BB is a good indicator of stratiform rain, BB is detected in only about $40 \%$ of stratiform rain. The BB count strongly depends on the antenna scan angle because of the smearing of BB shape, which becomes stronger as the antenna scan angle increases.

Comparison of HBB and the NCEP2 derived $0^{\circ} \mathrm{C}$ height HfreezeNCEP2 indicates that, on average, HBB appears about $500 \mathrm{~m}$ below HfreezeNCEP2.

Though there are some differences, the scatterplot of HBB versus HfreezeNCEP2 over water and that over land are similar, which implies that the NCEP2 temperature data are good for estimating HBB both over water and over land.

It was demonstrated that the application of a very simple filter can eliminate the BB outliers to a great extent. We are planning to introduce a similar but refined filter in 2A23 V7.

Analysis data, such as GANAL, NCEP, and NCEP2, may also be used to estimate the $0^{\circ} \mathrm{C}$ in $2 \mathrm{~A} 23$ V7. We are studying the possibility of using GANAL in $2 \mathrm{~A} 23 \mathrm{~V} 7$.

The effect of the boost of the TRMM satellite to a higher altitude appears in long-term statistics of rain type and BB. The boost has at least five impacts, (I1 to I5), on the data.

I1 Decreased detection of smaller Z after the boost because of slightly decreased radar sensitivity.

I2 Decreased measured $\mathrm{Z}$ after the boost because of increased scattering volume, which strengthens the NUF effect.

I3 Decreased detection of shallow rain near scan edges after the boost because of increased smearing of the surface clutter and the decreased detection of 
$\mathrm{BB}$ near the scan edges after the boost due to the increased smearing of the BB peak profile.

I4 Enhancement of impact I3 because of the increased footprint size after the boost.

I5 A slightly increased ZmaxBB after the boost in a statistical sense because of the overlapped footprints along the satellite track.

The stratiform count decreased after the boost because of impacts I1, I2, I3, and I4. The convective count decreased after the boost because of impacts I2, I3, and I4. However, impacts I3 and I4 mainly affect shallow isolated rain, which is classified as convective in 2A23 V6. The BB count decreased after the boost because of impacts I2, I3, and I4. The effect of the boost on the data is small, but discernible.

The zonal mean of $\mathrm{ZmaxBB}$ over water is almost constant in latitude, but the zonal mean of ZmaxBB over land is slightly lower in regions above 20 degrees latitude in the northern hemisphere.

BBwidth at nadir increases as ZmaxBB increases. The zonal mean of BBwidth at nadir is almost constant in the latitude between $15^{\circ} \mathrm{S}$ and $15^{\circ} \mathrm{N}$, but when the latitude becomes higher than $15^{\circ} \mathrm{S}$ or $15^{\circ} \mathrm{N}$, BBwidth at nadir decreases very slightly but almost linearly with respect to latitude.

\section{Acknowledgments}

This work was supported by the Japan Aerospace Exploration Agency (JAXA). TRMM products were obtained from JAXA and from the National Aeronautics and Space Administration (NASA). Some long-term statistical data on 2A23 were provided by the Remote Sensing Technology Center of Japan (RESTEC). NCEP2 data were obtained from the Web site of NOAA/OAR/ESRL PSD, Boulder, Colorado, USA.

\section{Appendix A Definition of BBwidth}

The width of BB (BBwidth) was computed from the upper and lower boundaries of $\mathrm{BB}$, which are defined as follows (see Fig. A1).

The lower boundary of BB (BBbottom) is defined as the range at which the greatest change in the slope of $\mathrm{Z}$ occurs in the lower part of BB. This definition is very close to that by Fabry and Zawadzki (1995).

To determine the upper boundary of BB (BBtop), a quantity called Zrain is introduced. Zrain is defined as the value of $\mathrm{Z} 125 \mathrm{~m}$ below BBbottom because oversampled data with a $125 \mathrm{~m}$ resolution is used in the computation.

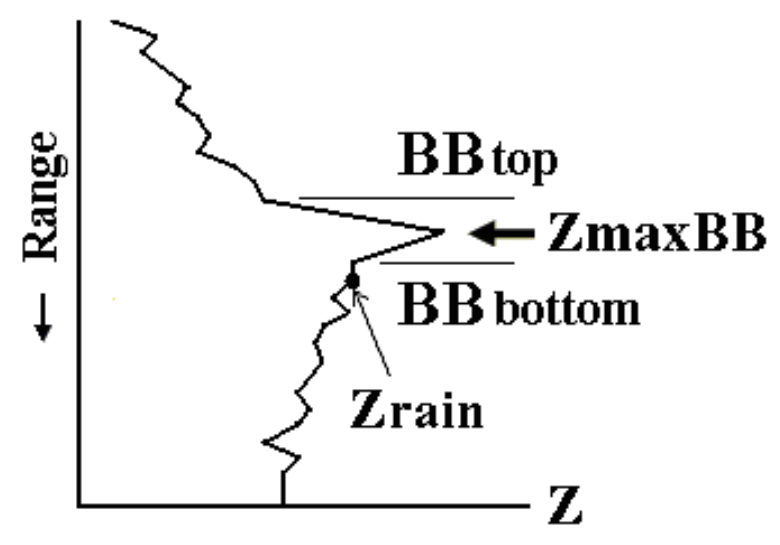

Fig. A1. Schematic illustration of BBtop, BBbottom, ZmaxBB, and Zrain.

The upper boundary of BB (BBtop) is determined by finding the following two Points.

$\mathrm{P} 1$ : The range at which the greatest in the slope of $\mathrm{Z}$ occurs in the region above the height of the BB peak, HBB. This search is made upward starting from the range corresponding to HBB.

$\mathrm{P} 2$ : The range at which $\mathrm{Z}$ becomes less than Zrain for the first time when $\mathrm{Z}$ is examined upward in the upper part of $\mathrm{BB}$ starting from the range corresponding to HBB.

When P1 and P2 are the same, BBtop is defined as the range of $\mathrm{P} 1$ (which is the same as that of $\mathrm{P} 2$ in this case). When P1 and P2 differ, however, the point closer to HBB is selected between P1 and P2, and BBtop is defined as the range of the selected point.

The above definition of BBtop differs from but somewhere between that given by Fabry and Zawadzki (1995) and by Klaassen (1988).

BBwidth at the nadir angle bin is computed simply by the following equation.

$$
\begin{aligned}
\text { BBwidth }= & (\text { range of BBbottom }) \\
& -(\text { range of BBtop })
\end{aligned}
$$

Though not used in this paper, we give the formula for computing BBwidth at angle bins other than the nadir angle bin for the sake of completeness of discussion. When the antenna beam points in other than the nadir direction, the upper and lower boundaries are determined in the same manner as in the case of nadir angle bin, but the effect of oblique incidence is subtracted by using the following empirical formula: 
BBwidth $=(($ range of BBbottom $)-($ range of BBtop $)$

$$
\left.-\frac{\mathrm{LoF}}{\cos ^{2} \theta} \sin \theta\right) \cos \theta
$$

where $\theta$ is the local zenith angle, Lo is the diameter of the antenna beam footprint, and $\mathrm{F}(<1)$ is an empirical correction factor.

\section{Appendix B Conditional probability}

This appendix briefly discusses the conditional probability and the unconditional probability.

Let us denote the quantity considered by $X$, and divide $X$ into bins having the same bin size and denote the representative value of the quantity at the i-th bin by $X_{i}$ and the count of $X_{i}$ by $n_{i}$. Let us also assume that the quantity considered is observed in the range bin number $i=1,2,3, \ldots, N$. The conditional mean of $X$ may then be obtained by the following formula.

$$
(\text { conditional mean of } X)=\left(\sum_{i=1}^{N} n_{i} X_{i}\right) /\left(\sum_{i=1}^{N} n_{i}\right) \text {. }
$$

The above averaging is conditional because the average is taken under the condition that the quantity $X$ is observed, or stated differently, the above average is taken using the value with $n_{i}>0$.

Equation (B1) can be rewritten as follows:

$$
\text { (conditional mean of } X)=\sum_{i=1}^{N} p_{i} X_{i} \text {, }
$$

where $p_{i}$ is the conditional probability for the $i$-th bin defined by

$$
p_{i} \equiv n_{i} /\left(\sum_{i=1}^{N} n_{i}\right)
$$

The conditional probability has the following property.

$$
\sum_{i=1}^{N} p_{i}=1
$$

For TRMM PR data, the unconditional mean can be defined in the following way:

$$
\begin{aligned}
\text { (unconditional mean of } X) & =\left(\sum_{i=1}^{N} n_{i} X_{i}\right) \\
& /\left(\sum_{i=1}^{N} n_{i}+n_{0}\right),
\end{aligned}
$$

where $n_{0}>0$ and $\sum_{i=1}^{N} n_{i}+n_{0}$ is the total number of pixels observed by the TRMM PR.

The unconditional probability $p_{i}^{u n c}$ for the $i$-th bin can be defined similarly:

$$
p_{i}^{u n c} \equiv n_{i} /\left(\sum_{i=1}^{N} n_{i}+n_{0}\right)
$$

The conditional probability is preferable for a quantitative study of a physical quantity. Think of the following extreme example that for some physical reason the quantity considered gives always almost the same observed value that falls in the same bin with $i$ $=I$ having the value $X_{I}$. In such a case, the conditional mean becomes $X_{I}$ as it should be. If, however, the unconditional mean gives a value that is less than $X_{I}$, which mean value also varies depending on the count $n_{0}$.

Equation (B4) is the key to understanding the behavior of the mean value of ZmaxBB in Fig. 6 and the percentage of the count of ZmaxBB in Fig. 10.

Let us consider a certain PR quantity $X$, which you may regard as ZmaxBB. Let us suppose that $X$ is divided into bins having values $X i(i=1,2, \ldots, N)$, which are arranged as $X_{1}<X_{2}<\ldots<X_{N}$. Let us express the conditional probability of $X$ before the boost by $p_{i}(i=1$, $2, \ldots, N)$, and that after the boost by $p_{i}^{\prime}(i=1,2, \ldots, N)$. Equation (B4) states that $\sum_{i=1}^{N} p_{i}=1$ and $\sum_{i=1}^{N} p_{i}^{\prime}=1$. Let us consider a very simple example in which only $p_{i}^{\prime}$ becomes smaller than $p_{1}$. Since $\sum_{i=1}^{N} p_{i}^{\prime}=1$, some other $p_{i}^{\prime}(i>1)$, single or plural, must exceed $p_{i}$, which shifts the shape of the probability towards a higher value after the boost and the conditional mean of $X$ becomes higher after the boost.

\section{Appendix C \\ Use of 3A25 V6 for a scatterplot of BBwidth versus $\mathrm{ZmaxBB}$}

The relation between BBwidth and ZmaxBB can 
be obtained by plotting the data in the form of a scatterplot. Let us denote BBwidth as $y$ and ZmaxBB as $x$. We then want to obtain a relation that may be expressed as

$$
y=f(x),
$$

where $f$ is the function that expresses the relation between BBwidth (i.e., $y$ ) and $\mathrm{ZmaxBB}$ (i.e., $x$ ).

The 2A23 V6 product gives data sets

$$
\left(x_{1}, y_{1}\right),\left(x_{2}, \mathrm{y}_{2}\right), \cdots,\left(x_{i}, y_{i}\right), \cdots,
$$

where the index $i$ runs $i=1,2,3, \ldots$ when data are linearly numbered. Since this paper discusses BBwidth obtained in the nadir direction, the observed $y_{i}$ (when expressed in units of $[\mathrm{m}]$ ) can only take discrete values of $125,250,375, \ldots$ When we plot these data, there are no intermediate values in the $y$ direction of the scatterplot.

The situation is different when the 3A25 V6 data are used. The 3A25 V6 data sets may be expressed as follows:

$$
\left(\bar{x}_{1}, \bar{y}_{1}\right),\left(\bar{x}_{2}, \bar{y}_{2}\right), \cdots,\left(\bar{x}_{j}, \bar{y}_{j}\right), \cdots
$$

where the index $j(j=1,2,3, \ldots)$ specifies the 3 A35 grid cell, and $\bar{x}_{j}$, and $\bar{y}_{j}$ are defined as

$$
\bar{x}_{j} \equiv \sum_{i} x_{i j} / n_{j}, \quad \bar{y}_{j} \equiv \sum_{i} y_{i j} / n_{j},
$$

and where $x_{i j}$ is $x_{i}$ in grid cell $j, y_{i j}$ is $y_{i}$ in grid cell $j$, and $n_{j}$ is the number of data in grid cell $j$.

Suppose that the function $f$ is linear with respect to its argument, then it follows that $\bar{y}_{j}=f\left(\bar{x}_{j}\right)$ provided that $y_{i}=f\left(x_{i}\right)$. If the function $f$ is linear, which is expected to be the case for the relation between BBwidth and $\mathrm{ZmaxBB}$, the scatterplot of $\bar{y}_{j}$ versus $\bar{x}_{j}$ gives us the information about the function $f$.

Figure 14 in the text presents a scatterplot of $\bar{y}_{j}$ and $\bar{x}_{j}$. Since $\bar{y}_{j}$ is obtained by the average of $y_{i j}$ in grid cell $j$, the quantity $\bar{y}_{j}$ (when expressed in units of [m]) can take intermediate values other than multiples of 125. The appearance of the intermediated values in the $y$ direction of Fig. 14 is very useful for visually obtaining the information about the relation between
BBwidth and ZmaxBB.

\section{References}

Amitai, E., 1999: Relationships between Radar properties at high elevations and surface rain rate: Potential use for spaceborne rainfall Measurements, J. Appl. Meteor., 38, 321-333.

Awaka, J., T. Iguchi, and K. Okamoto, 2007: Rain type classification algorithm, Measuring Precipitation from Space: EURAINSAT and the Future, V. Levizzani et al., Eds., 213-224.

Battan, L. J., 1973: Radar Observation of the Atmosphere, Univ. of Chicago Press, Chicago, Illinois, $324 \mathrm{pp}$.

Bringi, V. N., and V. Chandrasekar, 2001: Polarimetric Doppler Weather Radar, Cambridge Univ. Press, Cambridge, 636 pp.

Fabry, F., and I. Zawadzki, 1995: Long-term radar observations of the melting layer of precipitation and their interpretation, J. Atmos. Sci., 52, 838-851.

Foote, G. B., and P. S. du Toit, 1969: Terminal velocity of raindrops aloft, J. Apple. Meteor., 8, 249253.

Harris, G. N., Jr., K. P. Bowman, and D.-B. Shin, 2000: Comparison of NCEP reanalysis and TRMM Precipitation Radar estimates of melting-layer altitudes, J. Climate, 13, 4137-4148.

Iguchi, T., T. Kozu, R. Meneghini, J. Awaka, and K. Okamoto, 2000: Rain profiling algorithm for the TRMM Precipitation Radar, J. Appl. Meteor., 39, 2038-2052.

Klaassen, W., 1988: Radar observations and simulation of the melting layer of precipitation, $J$. Atmos. Sci., 45, 3741-3753.

Kobayashi, S., and T. Iguchi, 2003: Variable pulse repetition frequency for the Global Precipitation Measurement Project (GPM), IEEE Trans. Geosci. Remote Sens., 41, 1714-1718.

Kozu, T., T. Kawanishi, H. Kuroiwa, M. Kojima, K. Oikawa, H. Kumagai, K. Okamoto, M. Okumura, H. Nakatsuka, and K. Nishikawa, 2001: Development of Precipitation Radar onboard the Tropical Rainfall Measuring Mission (TRMM) satellite, IEEE Trans. Geosci. Remote Sens., 39, 102-116.

Kummerow, C., J. Simpson, O. Thiele, W. Barnes, A. T. C. Chang, E. Stocker, R. F. Adler, A. Hou, R. Kaker, F. Wentz, P. Ashcroft, T. Kozu, Y. Hong, K. Okamoto, T. Iguchi, H. Kuroiwa, E. Im, Z. Haddad, G. Huffman, B. Ferrier, W. S. Olson, 
E. Zipser, E. A. Smith, T. T. Wilheit, G. North, T. Krishnamurti, and K. Nakamura, 2000: The status of the Tropical Rainfall Measuring Mission (TRMM) after two years in orbit, $J$. Appl. Meteor., 39, 1965-1982.

Liu, C., E. J. Zipser, D. J. Cecil, S. W. Nesbitt, and S. Sherwood, 2008: A cloud and precipitation feature database from nine years of TRMM observations, J. Appl. Meteor. Climatol., 47, 2712 2728.

Marshall, J. S., and W. Palmer, 1948: The distribution of raindrops with size, J. Meteor., 5, 165-166.

Meneghini, R., and T. Kozu, 1990: Spaceborne Weather Radar, Artech House, Boston-London, $199 \mathrm{pp}$.

Okamoto, K., Y. Ishido, and R. Meneghini, 1998: TRMM Precipitation Radar algorithms, $J$. Remote Sens. Soc. Japan, 18, 40-51, (in Japanese).

Oki, R., and A. Sumi, 1994: Sampling simulation of TRMM rainfall estimation using RadarAMeDas composite, J. Appl. Meteor., 33, 15971608.
Schumacher, C., and R. A. Houze Jr., 2003: The TRMM Precipitation Radar's view of shallow, isolated rain, J. Appl. Meteor., 42, 1519-1524.

Steiner, M., R. A. Houze Jr., and S. Yuter, 1995: Climatological characterization of three-dimensional storm structure from operational radar and rain gauge data, J. Appl. Meteor., 34, 19782007.

Takahashi, N., and T. Iguchi, 2004: Estimation and correction of beam mismatch of the precipitation radar after an orbit boost of the tropical rainfall measuring mission satellite, IEEE Trans. Geosci. Remote Sens., 42, 2362-2369.

Thurai, M., E. Deguchi, K. Okamoto, and E. Salonen, 2005: Rain height variability in the Tropics, IEE Proc. - Microwaves, Antennas Propagation, 152, 17-23.

Yamamoto, M. K., A. Higuchi, and K. Nakamura, 2006: Vertical and horizontal structure of winter precipitation systems over the western Pacific around Japan using TRMM data, J. Geophy. Res., 111, D13108, doi:10.1029/2005JD006412. 